\title{
Early Detection of Parametric Roll Resonance on Container Ships
}

\author{
Galeazzi, Roberto; Blanke, Mogens; Poulsen, Niels Kjølstad
}

Published in:

I E E E Transactions on Control Systems Technology

Link to article, DOI:

10.1109/TCST.2012.2189399

Publication date:

2013

Link back to DTU Orbit

Citation (APA):

Galeazzi, R., Blanke, M., \& Poulsen, N. K. (2013). Early Detection of Parametric Roll Resonance on Container Ships. I E E E Transactions on Control Systems Technology, 21(2), 489-503.

https://doi.org/10.1109/TCST.2012.2189399

\section{General rights}

Copyright and moral rights for the publications made accessible in the public portal are retained by the authors and/or other copyright owners and it is a condition of accessing publications that users recognise and abide by the legal requirements associated with these rights.

- Users may download and print one copy of any publication from the public portal for the purpose of private study or research.

- You may not further distribute the material or use it for any profit-making activity or commercial gain

- You may freely distribute the URL identifying the publication in the public portal

If you believe that this document breaches copyright please contact us providing details, and we will remove access to the work immediately and investigate your claim. 


\title{
Early Detection of Parametric Roll Resonance on Container Ships
}

\author{
Roberto Galeazzi, Member, IEEE, Mogens Blanke, Senior Member, IEEE, \\ and Niels K. Poulsen, Senior Member, IEEE
}

\begin{abstract}
Parametric roll resonance on ships is a nonlinear phenomenon where waves encountered at twice the natural roll frequency can bring the vessel dynamics into a bifurcation mode and lead to extreme values of roll. Recent years have shown several incidents with dramatic damage on container vessels. The roll oscillation, sub-harmonic with respect to the wave excitation, may be completely unexpected and a system for detection of the onset of such resonance could warn the navigators before roll angles reach serious levels. Timely warning could make remedial actions possible, change of ship's speed and course, to escape from the bifurcation condition. This paper proposes non-parametric methods to detect the onset of roll resonance and demonstrates their performance. Theoretical conditions for parametric resonance are re-visited and are used to develop efficient methods to detect its onset. Spectral and temporal correlation of the square of roll with pitch (or heave) are demonstrated to be of particular interest as indicators. Properties of the indicators are scrutinized and a change detector is designed for the Weibull type of distributions that were observed from a time-domain indicator for phase correlation. Hypothesis testing for resonance is developed using a combination of detectors to obtain robustness. Conditions of forced roll and disturbances in real weather conditions are analyzed and robust detection techniques are suggested. The efficacy of the methodology is shown on experimental data from model tests and on data from a container ship crossing the Atlantic during a storm.
\end{abstract}

Index Terms-Likelihood ratio test, non-Gaussian distribution, parametric roll resonance, ship dynamic stability, spectral correlation test, statistical change detection.

\section{INTRODUCTION}

$\mathbf{P}$ ARAMETRIC roll is a nonlinear phenomenon that belongs to the category of autoparametric resonance, a type of resonance that takes place in systems characterized by periodic variations of some of their parameters. Parametric resonance occurs in different kinds of systems, from beneficial in micro-electro-mechanics where parametric excitation is induced on purpose to decrease the parasitic signal in capacitive sensing [1], to critical if occurring on motorcycles, where unstable steering oscillations may develop due to a cyclic varying road profile [2]. Observations of parametric resonance

Manuscript received December 28, 2010; revised October 20, 2011 and February 5, 2012.

This work was supported by the Danish Agency for Research and Innovation, the Joint Proof of Concept Project Fund, grant number FI 07-017766.

R. Galeazzi (corresponding author) and M. Blanke are with Department of Electrical Engineering, Technical University of Denmark, Kgs. Lyngby, Denmark, and with the Centre for Ships and Ocean Structures, Norwegian University of Science and Technology, Trondheim, Norway. r.galeazzi@ieee.org and mbelektro.dtu.dk

N. K. Poulsen is with Department of Informatics and Mathematical Modelling, Technical University of Denmark, Kgs. Lyngby, Denmark. nkp@imm.dtu.dk on ships were first done by Froude ([3], [4]) who reported that a vessel, whose frequency of oscillation in heave/pitch is twice its natural frequency in roll, shows undesirable seakeeping characteristics, which can lead to the possibility of exciting large roll oscillations. Parametrically induced roll has been on the agenda of the marine research community since the early 1950 's when stability issues related with possible large roll oscillations in longitudinal seas were addressed in [5] and [6]. However, it was only after the report by France et al. [7] about the root causes of the very significant losses suffered by the APL China container ship in October 1998, that parametric roll resonance reached the top of the priority list among the ships' stability related phenomena. Døhlie [8] emphasized parametric resonance as a very concrete phenomenon able to threaten some of the giants of the sea in common passage conditions, which were previously considered to be of no danger. Parametric roll resonance can occur at other ratios than $1: 2$ between the natural frequency of roll and the excitation, but this resonance is the one in focus in the marine industry as being a major risk to ships during passage.

The last decade has shown a significant number of publications devoted to parametric roll. Particular focus on container ships were given in [9]-[17]. Fishing vessels were in focus in [18], [19]. An overview of parametric resonance in mechanical systems appeared in [20] where several chapters are dedicated to parametric resonance on ships including [21], [22] and [23]. The topic at the core of the research has been to analyze the nonlinear interactions between roll and other of ship's motions in order to develop models, which could predict vessels' susceptibility to parametric roll already at the design stage. However, the large amount of ships already built, and newbuildings that take parametric resonance as a calculated risk, could not benefit from better models and new hull designs. These vessels could enhance their safety against parametric roll only through novel on-board decision support systems, which should be capable of giving an early warning of the onset of the phenomenon, such that navigators or automatic systems could counteract that parametric roll resonance develops to its full devastating magnitude.

For detection of onset of the bifurcation condition, Holden $e t$ al. [24] proposed an observer based predictor, which estimates the eigenvalues of a linear second-order oscillatory system. The algorithm issues a warning when those eigenvalues move into the right-half plane. The method works convincingly but it was designed to cope with excitation by narrow band regular waves. Irregular sea conditions were studied by [25] who used finite time Lyapunov exponents to detect the onset of 
parametric roll, but this method was not found to possess sufficient robustness when validated against experimental data. Some commercial products are also available, where a specific feature for the prediction of the risk of parametric roll is included in the decision support system. Those systems, named first generation warning systems by Døhlie [8], rely on longer horizon analysis of responses and provide polar diagrams with risk zones in speed and heading.

Starting from the early results outlined in [26] and [27] this paper re-visits the core of the theory of parametric resonance and proposes signal-based methods for detection of parametric roll. Then, it discusses how robustified detection could be obtained, since the promising detectors proposed in [26] and [27] could not achieve sufficiently reliable performance in real sea scenarios. Indicators for the onset of parametric roll are discussed, and through the use of statistical change detection tools, it is shown possible to obtain robust detection based solely on signals. Its core is shown to be two detection schemes: one in the frequency domain, a second in the time domain. The frequency-domain detector uses spectral correlation between pitch, directly excited by the waves, and the sub-harmonic roll motion. The time-domain detector exploits phase synchronization that occurs in parametric resonating systems, using correlation between the square of the roll and pitch. A driving signal carrying the phase information is designed, and it is shown to exhibit an amplitude distribution following a double Weibull distributed process. A generalized likelihood ratio test (GLRT) is then set up. Sensitivity to usual forced roll from waves is analyzed and the detection system's performance is evaluated. Two data sets are used: experimental data from towing tank tests and motion data from a container ship passage in heavy seas over the Atlantic.

The paper is structured as follows: Section II introduces parametric roll in a descriptive manner to provide physical insight and the phenomenon is then formalized revisiting the framework of autoparametric systems. Section III suggests two detection methods, and offers analytical derivation of the detection indexes. Section IV presents the engineering of the monitoring system with focus on robustification of the detection methods. Section V offers a thorough analysis of the performance of the warning system tested on model scale and full scale data sets. Section VI draws some conclusions.

\section{PARAMETRIC ROLL - CONDITIONS AND UNDERLYING PHYSICS}

This section presents empirical experience and introduces a mathematical treatment of parametric roll resonance.

\section{A. Empirical Experience}

Empirical conditions have been identified that may trigger parametric roll resonance when the roll dynamics is in the principal parametric resonance region:

1) the period of the encounter wave is approximately equal to half the roll natural period $\left(T_{e} \approx \frac{1}{2} T_{\phi}\right)$

2) the wave length and ship length are approximately equal $\left(\lambda_{w} \approx L_{\mathrm{PP}}\right)$

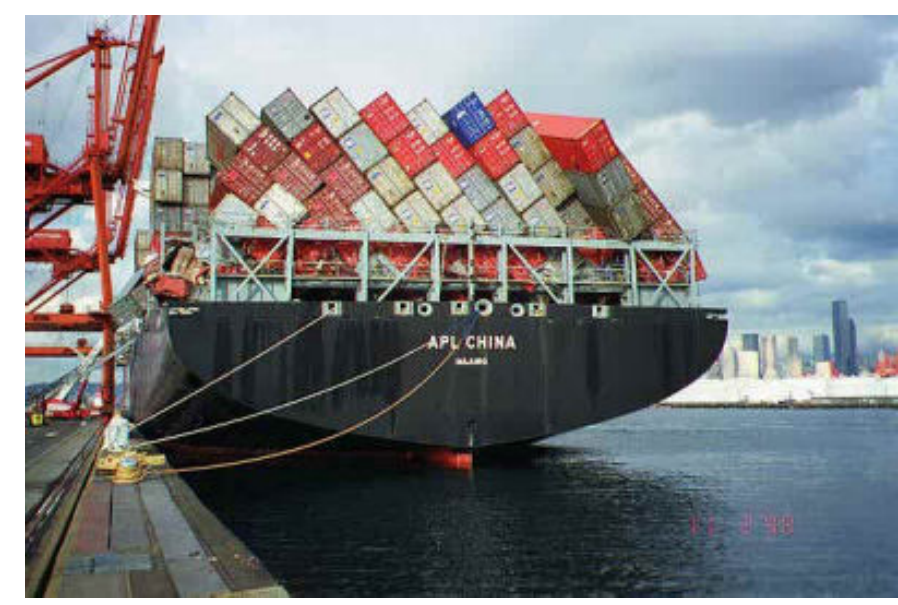

Fig. 1. Devastation of on-deck containers after the APL China underwent parametric roll resonance. (http://www.cargolaw.com/)

3) the wave height is greater than a ship-dependent threshold $\left(h_{w}>\bar{h}_{s}\right)$

4) the ship's roll damping is low.

When those conditions are met, and the ship sails in moderate to heavy longitudinal or oblique seas, then the wave passage along the hull and the wave excited vertical motions result in variations of the underwater hull geometry, which in turn change the roll restoring characteristics. The onset of parametric resonance causes a sudden and quick rise of roll oscillation, which can reach amplitudes larger than $\pm 40^{\circ}$ ([7], [28]), and it may bring the vessel into conditions dangerous for the cargo, the crew and the hull integrity. An impression of the damages produced by parametric roll is provided in Fig. 1, which shows the devastation suffered by APL China - the damage exceeded the value of the vessel itself [29].

\section{B. Mathematical Formulation}

To capture the sparkle that induces the development of parametric roll, consider a vessel sailing at constant forward speed in moderate head regular seas. The incident wave gives rise to forces and moments acting on the hull. In head regular seas, conventional forced roll cannot occur since forces and moments from wave pressure on the hull have no lateral components; only motions in the vertical plane can be excited. Heaving and pitching cause periodic variations of the submerged hull geometry. During a wave passage, the underwater volume of the vessel changes from the still water case, resolving in a variation of the position of the center of buoyancy, but, most important, in a variation of the metacentric radius [30]. This in turn gives rise to a modification of the transverse metacentric height GM and also to a new position of the metacentre M. Consequently the periodic fluctuation of GM, which can be considered sinusoidal,

$$
\mathrm{GM}(t)=\overline{\mathrm{GM}}+\mathrm{GM}_{\mathrm{a}} \cos \omega_{e} t
$$

influences the stability properties of the vessel through the roll restoring moment that is approximated by

$$
\tau(t) \approx \rho g \nabla \mathrm{GM}(t) \sin \phi
$$


where $\overline{\mathrm{GM}}$ is the mean value of the metacentric height, $\mathrm{GM}_{\mathrm{a}}$ is the amplitude of the variations of the metacentric height in waves, $\rho$ is the water density, $g$ is the acceleration of gravity, and $\nabla$ is the displaced volume. In still water $\operatorname{GM}(t)=\overline{\mathrm{GM}}$, hence $\tau(t)=\tau_{0}$. In waves, these two situations alternate:

- wave trough amidships: $\operatorname{GM}(t)>\overline{\mathrm{GM}}$ causing a larger restoring moment $\left(\tau>\tau_{0}\right)$ and increased stability

- wave crest amidships: $\operatorname{GM}(t)<\overline{\mathrm{GM}}$ hence a smaller restoring moment $\left(\tau<\tau_{0}\right)$ and reduced stability.

This alternate sequence of instantaneous increased and reduced restoring moment causes the roll angle to keep increasing unless some other factors start counteracting it.

Formally, this can be described as the interaction between coupled modes of an autoparametric system, where the primary system is externally forced by a sinusoidal excitation. Starting from the three degrees-of-freedom $3^{\text {rd }}$ order nonlinear heave-pitch-roll model presented in [31], a simplified two degrees-of-freedom pitch-roll model is exploited to analyse the onset of parametric roll resonance. This model is obtained by neglecting the heave interaction with roll, and by limiting the analysis to first order wave/hull interaction.

Let $\theta$ be the pitch angle, and $\phi$ the roll angle, then the two degrees-of-freedom pitch-roll model is,

$$
\begin{aligned}
\left(I_{y}+M_{\ddot{\theta}}\right) \ddot{\theta} & +M_{\dot{\theta}} \dot{\theta}+M_{\theta} \theta+\frac{1}{2}\left(M_{\phi \phi} \phi^{2}+M_{\theta \theta} \theta^{2}\right) \\
& +\frac{1}{6}\left(M_{\theta \theta \theta} \theta^{3}+3 M_{\phi \phi \theta} \phi^{2} \theta\right)+M_{\zeta \theta}(t) \theta \\
& =M_{\mathrm{ext}} \cos \left(\omega_{e} t+\psi\right) \\
\left(I_{x}+K_{\ddot{\phi}}\right) \ddot{\phi} & +K_{\dot{\phi}} \dot{\phi}+K_{\dot{\phi}|\dot{\phi}|} \dot{\phi}|\dot{\phi}|+K_{\phi} \phi+K_{\phi \theta} \phi \theta \\
& +\frac{1}{6}\left(K_{\phi \phi \phi} \phi^{3}+3 K_{\theta \theta \phi} \theta^{2} \phi\right) \\
& +K_{\zeta \phi}(t) \phi=0
\end{aligned}
$$

where the pitch dynamics (1) is the primary system externally forced, and the roll dynamics (2) is the secondary system, nonlinearly coupled with the primary, and which is initially at rest. The model coefficients are:

- Rigid body inertias: $I_{x}$ in roll, $I_{y}$ in pitch

- Added inertias: $K_{\ddot{\phi}}, M_{\ddot{\theta}}$ in roll and pitch (a positive sign convention is used here for added inertia following the notation by Newman [30], several other authors in hydrodynamic literature use a negative sign convention)

- Damping: $K_{\dot{\phi}}, M_{\dot{\theta}}$ are coefficients for linear part; $K_{\dot{\phi}|\dot{\phi}|}$ is for quadratic damping

- Restoring moments due to body motion: $K_{\phi}, K_{\phi \theta}, K_{\phi \phi \phi}$, $K_{\theta \theta \phi}$ are the coefficients of the roll restoring moment; $M_{\theta}, M_{\phi \phi}, M_{\theta \theta}, M_{\theta \theta \theta}, M_{\phi \phi \theta}$ are the coefficients of the pitch restoring moment

- Restoring moments due to wave/hull interaction: $K_{\zeta \phi}(t)$, $M_{\zeta \theta}(t)$ and are functions of wave amplitude $\zeta(t)$

- External moments due to direct wave action: $M_{\mathrm{ext}}$ is the amplitude of the wave-induced pitch moment, and $\omega_{e}$ is the wave encounter frequency.
System (1)-(2) can be rewritten as

$$
\begin{aligned}
& \ddot{\theta}+\nu_{1} \dot{\theta}+\left(\omega_{\theta}^{2}+\mu_{5}(t)+\mu_{4} \phi^{2}\right) \theta \\
& \quad+\mu_{1} \phi^{2}+\mu_{2} \theta^{2}+\mu_{3} \theta^{3}=f \cos \left(\omega_{e} t+\psi\right) \\
& \ddot{\phi}+\nu_{2} \dot{\phi}+\nu_{22} \dot{\phi}|\dot{\phi}| \\
& \quad+\left(\omega_{\phi}^{2}+\kappa_{4}(t)+\kappa_{1} \theta+\kappa_{2} \theta^{2}\right) \phi+\kappa_{3} \phi^{3}=0
\end{aligned}
$$

with coefficients

$$
\begin{aligned}
& \nu_{1}=\frac{M_{\dot{\theta}}}{I_{y}+M_{\ddot{\theta}}} \quad \nu_{2}=\frac{K_{\dot{\phi}}}{I_{x}+K_{\ddot{\phi}}} \quad \nu_{22}=\frac{K_{\dot{\phi}|\dot{\phi}|}}{I_{x}+K_{\ddot{\phi}}} \\
& \omega_{\theta}=\sqrt{\frac{M_{\theta}}{I_{y}+M_{\ddot{\theta}}}} \quad \omega_{\phi}=\sqrt{\frac{K_{\phi}}{I_{x}+K_{\ddot{\phi}}}} \quad f=\frac{M_{\mathrm{ext}}}{I_{y}+M_{\ddot{\theta}}} \\
& \mu_{1}=\frac{1}{2} \frac{M_{\phi \phi}}{I_{y}+M_{\ddot{\theta}}} \quad \mu_{2}=\frac{1}{2} \frac{M_{\theta \theta}}{I_{y}+M_{\ddot{\theta}}} \quad \mu_{3}=\frac{1}{6} \frac{M_{\theta \theta \theta}}{I_{y}+M_{\ddot{\theta}}} \\
& \mu_{4}=\frac{1}{2} \frac{M_{\phi \phi \theta}}{I_{y}+M_{\ddot{\theta}}} \quad \mu_{5}(t)=\frac{M_{\zeta \theta}(t)}{I_{y}+M_{\ddot{\theta}}} \quad \kappa_{1}=\frac{K_{\phi \theta}}{I_{x}+K_{\ddot{\phi}}} \\
& \kappa_{2}=\frac{1}{2} \frac{K_{\theta \theta \phi}}{I_{x}+K_{\ddot{\phi}}} \quad \kappa_{3}=\frac{1}{6} \frac{K_{\phi \phi \phi}}{I_{x}+K_{\ddot{\phi}}} \quad \kappa_{4}(t)=\frac{K_{\zeta \phi}(t)}{I_{x}+K_{\ddot{\phi}}} .
\end{aligned}
$$

The semi-trivial solution of the system (3)-(4) is found by applying the multiple scales method [32] under the assumptions

- $f=O(1)$, that is large pitch responses can be generated also away from the pitch resonance condition $\omega_{e} \approx \omega_{\theta}$

- $\nu_{1}=O(1)$, that is the pitch response is bounded even at pitch resonance

- $\mu_{i}=O(\varepsilon), i=1, \ldots, 5$, that is the static and dynamic restoring terms have a small effect on the pitch response.

To first order approximation the semi-trivial solution at steady state is

$$
\begin{aligned}
\theta_{0}(t ; \varepsilon) & =F \cos \left(\omega_{e} t+\xi\right)+O(\varepsilon) \\
\phi_{0}(t) & =0
\end{aligned}
$$

where

$$
F=\frac{f}{\sqrt{\left(\omega_{\theta}^{2}-\omega_{e}^{2}\right)^{2}+\omega_{e}^{2} \nu_{1}^{2}}}, \quad \xi=\arctan \left(-\frac{\omega_{e} \nu_{1}}{\omega_{\theta}^{2}-\omega_{e}^{2}}\right) .
$$

The stability of the semi-trivial solution is investigated by looking at its behavior in a neighborhood defined as

$$
\begin{aligned}
& \theta(t)=\theta_{0}(t)+\delta_{\theta}(t) \\
& \phi(t)=\phi_{0}(t)+\delta_{\phi}(t),
\end{aligned}
$$

where $\delta_{\theta}$ and $\delta_{\phi}$ are small perturbations. By linearizing the system (3)-(4) around the semi-trivial solution, the following system is obtained in the perturbation variables $\delta_{\theta}$ and $\delta_{\phi}$

$$
\begin{aligned}
& \ddot{\delta}_{\theta}+\nu_{1} \dot{\delta}_{\theta}+\left(\omega_{\theta}^{2}+\mu_{5}(t)\right) \delta_{\theta}=0 \\
& \ddot{\delta}_{\phi}+\nu_{2} \dot{\delta}_{\phi} \\
& \quad+\left(\omega_{\phi}^{2}+\kappa_{4}(t)+\kappa_{1} \theta_{0}(t)+\kappa_{2} \theta_{0}^{2}(t)\right) \delta_{\phi}=0 .
\end{aligned}
$$

The system (9)-(10) consists of two decoupled parametrically excited equations, where the parametric excitations $\mu_{5}(t)$ and $\kappa_{4}(t)$ drive the respective system at $\omega_{e}$

$$
\begin{aligned}
& \mu_{5}(t)=\tilde{\mu}_{5} \cos \left(\omega_{e} t\right) \\
& \kappa_{4}(t)=\tilde{\kappa}_{4} \cos \left(\omega_{e} t\right) .
\end{aligned}
$$

Due to the physical and geometrical properties of surface vessels the pitch and roll natural frequencies are not equal; hence (9) and (10) cannot be in the principal parametric resonance 
condition at the same time. Conversely, it is plausible that the two subsystems are simultaneously into one of the instability regions characterizing parametrically excited systems.

According to stability theory [33], to trigger parametric resonance the parametric excitation must be larger than twice the linear damping. However, differently from the roll mode, the pitch mode is very well damped; hence this possibility can be discarded. For the considered model $\nu_{1} / \nu_{2} \approx 20$, and $\tilde{\mu}_{5} / \nu_{1} \approx 0.1$.

However, for marine craft, the condition $\omega_{e} \approx \omega_{\theta} \approx 2 \omega_{\phi}$ could also be relevant, which addresses a case where the pitch motion is in the fundamental parametric resonance region, while being also in the $1: 1$ resonance with the wave moment, and the roll motion is in the principal parametric resonance region. This case was not included in common analysis where the pitch dynamics was described linearly [34], or the timevarying component $M_{\zeta \theta}(t)$ due to wave/hull interaction had been neglected [35].

Under the assumption $\nu_{1} \gg \tilde{\mu}_{5}$ then (9) has solution $\delta_{\theta}=0$, which is asymptotically stable. Therefore the stability of the semi-trivial solution is fully determined by (10), which is the damped Hill equation, where the periodic coefficient (period $\left.T=2 \pi / \omega_{e}\right)$ up to first order approximation is

$$
\begin{aligned}
p(t)= & \omega_{\phi}^{2}+\kappa_{1}\left(F \cos \left(\omega_{e} t\right)\right)+\kappa_{2}\left(F \cos \left(\omega_{e} t\right)\right)^{2} \\
& +\tilde{\kappa}_{4} \cos \left(\omega_{e} t+\chi\right)
\end{aligned}
$$

where the phase shift $\xi$ has been neglected. Rewriting the periodic function as sum of complex exponentials it is possible to highlight the terms responsible for the onset of parametric resonance, i.e.

$$
\begin{aligned}
p(t)= & \omega_{\phi}^{2}+\frac{1}{2} \kappa_{2} F^{2}+\frac{1}{2}\left(\kappa_{1} F+\tilde{\kappa}_{4}\right) e^{j \omega_{e} t} \\
& +\frac{1}{2}\left(\kappa_{1} F+\tilde{\kappa}_{4}\right) e^{-j \omega_{e} t}+\text { h.f.t. }
\end{aligned}
$$

where h.f.t. are higher frequency terms, whose frequencies are not in the ratio $1: 2$ with the roll natural frequency.

The solution of (10) if found applying the method of multiple scales [32] under the assumptions

- $\nu_{2}=O(\varepsilon), \kappa_{1}=O(\varepsilon), \tilde{\kappa}_{4}=O(\varepsilon)$, that is the linear roll damping is of the same order of magnitude of the parametric excitation $\kappa_{1} F+\tilde{\kappa}_{4}$

- $\kappa_{2}=O\left(\varepsilon^{2}\right), \kappa_{3}=O\left(\varepsilon^{2}\right)$, that is the higher order roll restoring components have a small influence on the roll response.

To first order approximation the roll response is

$$
\phi(t ; \varepsilon)=\alpha(\varepsilon t) \cos \left(\frac{1}{2} \omega_{e} t-\chi(\varepsilon t)\right)+O(\varepsilon),
$$

where $\alpha(\varepsilon t)$ is an exponential envelope which grows or decays with time depending on the magnitude of the parametric excitation and the linear damping coefficient of the roll subsystem. Application of Floquet theory [33] allows to determine the boundaries of the principal instability region of (10), which are

$$
\frac{1}{4} \frac{\nu_{2}^{2}}{\omega_{e}^{2}}+\left(\frac{\omega_{\phi}^{2}}{\omega_{e}^{2}}-\frac{1}{4}\right)^{2}=\frac{1}{4} \frac{\eta^{2}}{\omega_{e}^{4}},
$$
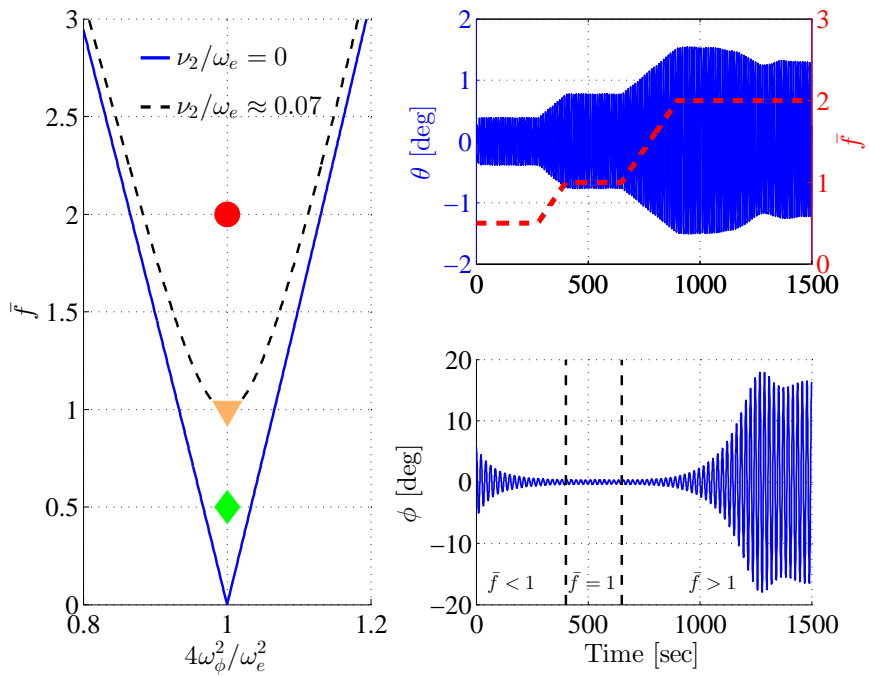

Fig. 2. Parametric resonance in the pitch-roll autoparametric system: (left) stability diagram of the secondary system for different levels of damping, and different amplitude of the excitation; (right) pitch and roll time series evolution for increasing level of the external excitation.

where

$$
\eta=\frac{\kappa_{1} f+\tilde{\kappa}_{4} \sqrt{\left(\omega_{\theta}^{2}-\omega_{e}^{2}\right)^{2}+\omega_{e}^{2} \nu_{1}^{2}}}{\sqrt{\left(\omega_{\theta}^{2}-\omega_{e}^{2}\right)^{2}+\omega_{e}^{2} \nu_{1}^{2}}}
$$

is the amplitude of the parametric excitation. The boundary condition (16) can be used to determine the critical value $f_{c}$ of the external excitation, which triggers the parametric resonance in the secondary system, that is

$$
f_{c}=\frac{\sqrt{\left(\omega_{\theta}^{2}-\omega_{e}^{2}\right)^{2}+\omega_{e}^{2} \nu_{1}^{2}} \times}{\left[2 \omega_{e}^{2} \sqrt{\frac{1}{4} \frac{\nu_{2}^{2}}{\omega_{e}^{2}}+\left(\frac{\omega_{\phi}^{2}}{\omega_{e}^{2}}-\frac{1}{4}\right)^{2}}-\tilde{\kappa}_{4}\right] .}
$$

For $0<f<f_{c}$ the semi-trivial solution is stable and the pitch amplitude grows linearly with $f$. When $f>f_{c}$ then the semi-trivial solution loses stability and a non trivial solution in roll (15) appears.

The system (3)-(4) shows a saturation phenomenon both in pitch and in roll. In particular, when the excitation amplitude crosses the critical value, the amount of energy stored in the primary system stays constant and the entire energy rise flows into the secondary system. The rate at which energy is pumped into the secondary system varies according to the change of the phase $\chi$. When the rate at which energy being dissipated by viscous effects has matched the rate at which energy is transferred to the roll subsystem, the system reaches a steady state motion characterized by a constant amplitude and a phase shift $\chi=\pi$ (see Appendix A). Fig. 2 shows the development of parametric roll resonance while the amplitude of the excitation $\bar{f}=f / f_{c}$ increases: the stability chart clearly illustrates, in the parameter space, how the stability properties of the secondary system changes in response to a variation of the amplitude of the external excitation. 
Concluding, parametric roll is a resonance phenomenon triggered by the existence of the frequency coupling $\omega_{e} \approx 2 \omega_{\phi}$, and whose response shows a phase synchronization of $180^{\circ}$ with the parametric excitation.

\section{DETECTION METHODS}

The mathematical analysis of the development of parametric resonance in roll pointed out that the existence of this instability phenomenon is related to the simultaneous happening of two events: the wave encounter frequency is approximately twice the roll natural frequency, i.e. the roll motion is subharmonic with respect to the wave excitation; the pitch and roll motion show synchronization, i.e. every second peak of pitch is in-phase with a peak of roll.

Through the analysis of an experimental data set it is shown that these two conditions are also met in practice. Once these signals have been introduced, two complementary detection schemes are derived. One detection method works in the frequency domain and it is responsible of detecting the occurrence of the frequency coupling $\omega_{e} \approx 2 \omega_{\phi}$; the other works in the time domain and it searches for minimum synchronization level between pitch and roll.

\section{A. Indicators of Parametric Roll Resonance}

1) Frequency Coupling: In Section II it was shown that the onset and development of parametric roll is characterized by a sub-harmonic regime of the roll motion, which is featured by oscillations at half the wave frequency. In particular, the pitch mode, directly excited by the wave motion, pumps energy into the roll mode at a frequency about twice the roll natural frequency. Therefore when roll enters into parametric resonance an indication of the sub-harmonic regime could be obtained by looking at the evolution of the power spectrum of the square of the roll motion, since $\phi^{2}(t)$ should reveal an increasing amount of energy nearby the frequencies where pitch is transferring energy into roll. This is confirmed by the power spectra of $\phi(t), \phi^{2}(t)$, and $\theta(t)$ plotted in Fig. 3.

Given two discrete time signals $x[n]$ and $y[n]$, the similarity of the two waveforms is expressed through their crosscorrelation and cross-spectrum,

$$
\begin{aligned}
& \mathcal{R}_{x y}[m] \triangleq \sum_{m=-\infty}^{\infty} \bar{x}[m] y[n+m] \\
& \mathcal{P}_{x y}(\omega) \triangleq \sum_{m=-\infty}^{\infty} \mathcal{R}_{x y}[m] e^{-j \omega m}
\end{aligned}
$$

where $m$ is the time lag, and $\bar{x}$ is the complex conjugate of $x$. The magnitude of the cross-spectrum describes which frequency components of $x[n]$ are associated with large or small amplitudes at the same frequency of $y[n]$. Since the frequency components carrying useful information about the onset of parametric roll resonance are those about twice the roll natural frequency, the detection problem can be set up as monitoring the cross-spectrum of $\phi^{2}[n]$ and $\theta[n]$.
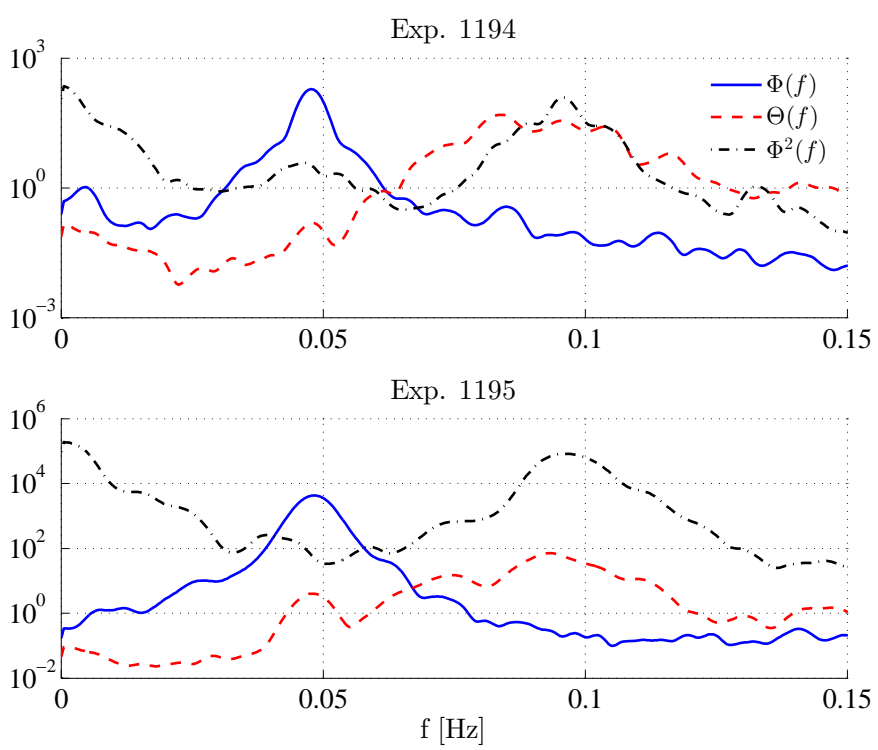

Fig. 3. Power spectra of $\phi(t), \phi^{2}(t)$, and $\theta(t)$ for the experiment 1194 where parametric roll did not occur and for the experiment 1195 where it occurred.

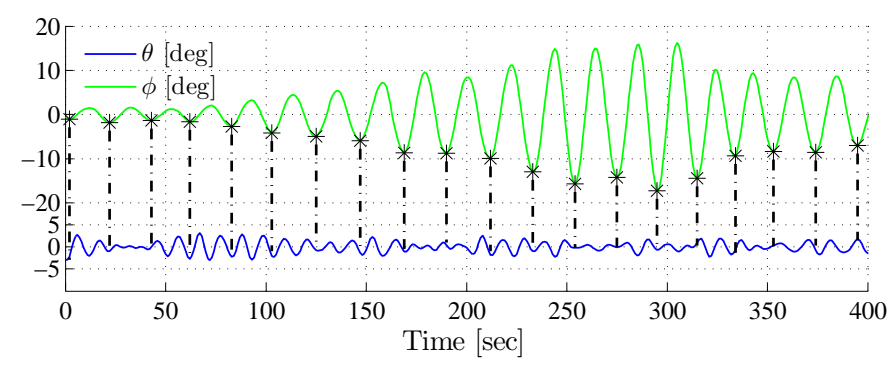

Fig. 4. Experiment 1195: alignment of peaks between pitch $\theta$ and roll $\phi$ during the onset and development of parametric roll.

2) Phase Synchronization: After onset, parametric roll resonance is characterized by non-linear synchronization between motions. Døhlie [8] pointed out that when parametric roll develops, there is a lining up of peaks between the pitch motion and the roll motion, such that every second peak of pitch is in-phase with the peak in roll. This is illustrated in Fig. 4 on model tank data where parametric roll resonance occurred. Fig. 4 also shows that when this alignment is partially lost, the roll oscillations starts decaying, seen between 150 and 250 seconds, or after 300 seconds in the plot. Therefore, a signal that carries the phase information of pitch and roll could be exploited for detection.

Consider two sinusoidal signals $s_{1}$ and $s_{2}$ given by

$$
\begin{aligned}
& s_{1}=A_{1} \cos \left(\omega t+\psi_{1}\right) \\
& s_{2}=A_{2} \cos \left(2 \omega t+\psi_{2}\right) .
\end{aligned}
$$

The square of $s_{1}$ is also a sinusoidal signal centered about $2 \omega$

$$
s_{1}^{2}=\frac{1}{2} A_{1}^{2}+\frac{1}{2} A_{1}^{2} \cos \left(2 \omega t+2 \psi_{1}\right)
$$


Multiplying $s_{1}^{2}$ by $s_{2}$ the following signal is obtained

$$
\begin{aligned}
s_{1}^{2} s_{2}= & \frac{1}{2} A_{1}^{2} A_{2} \cos \left(2 \omega t+\psi_{2}\right) \\
& +\frac{1}{2} A_{1}^{2} A_{2} \cos \left(2 \omega t+2 \psi_{1}\right) \cos \left(2 \omega t+\psi_{2}\right),
\end{aligned}
$$

which, by change of variable $\epsilon=2 \omega t+\psi_{2}$, is rewritten as

$$
s_{1}^{2} s_{2}=\frac{1}{2} A_{1}^{2} A_{2}\left(\cos \epsilon+\cos \left(\epsilon+2 \psi_{1}-\psi_{2}\right) \cos \epsilon\right) .
$$

Equation (24) shows that if $s_{1}^{2}$ and $s_{2}$ are in-phase $\left(2 \psi_{1}-\right.$ $\left.\psi_{2}=0\right)$ then

$$
s_{1}^{2} s_{2}=\frac{1}{2} A_{1}^{2} A_{2}\left(\cos \epsilon+\cos ^{2} \epsilon\right)
$$

whereas if $s_{1}^{2}$ and $s_{2}$ are out-of-phase $\left(2 \psi_{1}-\psi_{2}=\pi\right)$ then

$$
s_{1}^{2} s_{2}=\frac{1}{2} A_{1}^{2} A_{2}\left(\cos \epsilon-\cos ^{2} \epsilon\right),
$$

that is, the signal $s_{1}^{2} s_{2}$ shows positive or negative peaks of maximum amplitude $A_{1}^{2} A_{2}$.

Given the roll angle $\phi$ and the pitch angle $\theta$, we define the driving signal indicating the occurrence of parametric resonance in roll as

$$
d(t) \triangleq \phi^{2}(t) \theta(t) .
$$

The sign convention for roll and pitch is in agreement with [36], that is roll is positive for starboard side down and pitch is positive for bow up.

Consider Fig. 5, where $\phi(t)$ and $d(t)$ are plotted for one experiment without parametric roll (Exp. 1194) and another with parametric roll (Exp. 1195). The driving signal $d(t)$ appears to characterize quite well the amplitude variations of $\phi$ : when the amplitude of $\phi$ abruptly grows, a sequence of negative spikes shows up in the driving signal. In contrast, when the amplitude of $\phi$ decreases, positive spikes reflect this in $d(t)$. Moreover, when roll is in parametric resonance, the magnitudes of the negative spikes in the driving signal are much larger than those seen when the roll mode is not in a resonant condition. Therefore, a significant change in the variance of the driving signal $d(t)$ can be expected when parametric roll is developing. This jump in variance can be exploited by detection of change of signal power in the driving signal. To select the most appropriate detection scheme it is important to know the statistics of the signal.

The cumulative distribution functions (CDFs) plots for the non-resonant and the resonant cases (Fig. 6) indicate that the double Weibull PDF follows the behavior of the data much closer than either of Gaussian $(\mathcal{N})$ and Laplacian $(\mathcal{L})$ PDF. The two latter cut off the tails, and in particular the negative one, which is the most essential as it is an indicator of the presence of motion synchronization, hence denoting parametric roll resonance. The double Weibull distribution very well approximates the behavior of the negative tail, but shows some difficulty in trailing the positive tail. This could suggest that a non symmetric double Weibull distribution could be used, if return from resonance to non-resonance should be more accurately detected. Here, onset detection has the priority
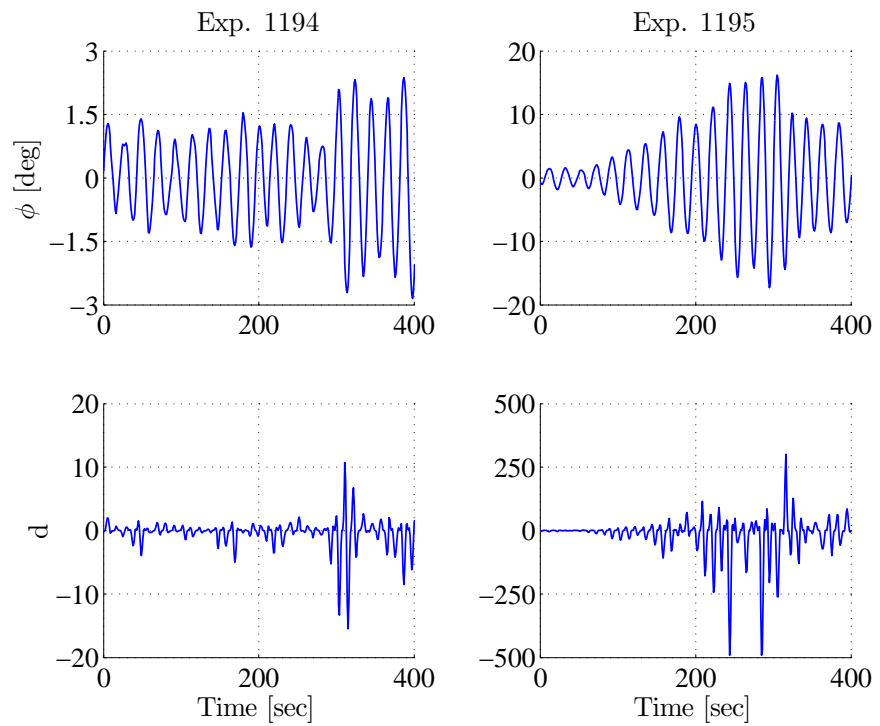

Fig. 5. Negative and positive peaks in $d$ address how the amplitude of the roll oscillations increases and decreases. Data from model basin test. Note the different $y$-axis range in the plots of the driving signal $d$.
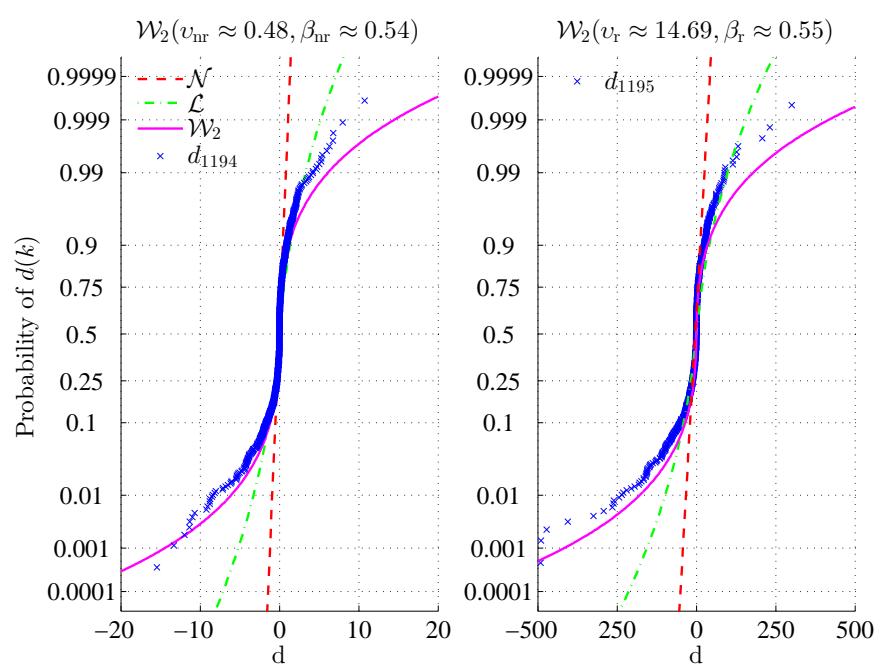

Fig. 6. Probability plots for a non resonant case (left), and a resonant case (right). Model basin test results. Three CDFs are fitted against the data: Gaussian $(\mathcal{N})$, Laplacian $(\mathcal{L})$, and double Weibull $\left(\mathcal{W}_{2}\right)$.

and the double Weibull PDF was chosen to describe the driving signal,

$$
p(d) \triangleq \mathcal{W}_{2}(d)=\frac{\beta}{2 v^{\beta}}|d|^{\beta-1} \exp \left(-\left(\frac{|d|}{v}\right)^{\beta}\right)
$$

where $v$, and $\beta$ are the scale and shape parameters.

A good discriminant between resonant and non resonant cases is the variation in signal power. Ideally, in the non resonant case the variance of $d(t)$ would be zero because no roll motion is expected when a ship sails in head seas. In real sailing conditions, instead, the wave train approaches the ship with a certain spreading factor, resulting in an excitation also along the transversal plane of the ship. This excitation induces roll oscillations, which in turn give a driving signal with power 
different from zero. Therefore, a detector that looks for abrupt changes in signal variance (power) is aimed at. The variance of a double Weibull distributed signal is given by

$$
\sigma^{2}=v^{2}\left[\Gamma\left(1+\frac{2}{\beta}\right)-\Gamma^{2}\left(1+\frac{1}{\beta}\right)\right]
$$

hence the detection scheme must trail variations in scale and shape parameters.

\section{B. Frequency Condition - Spectral Correlation Test}

The frequency condition to make a resonance possible with a 1 : 2 ratio between roll and pitch motions requires that spectral contents are present in roll at around the natural roll frequency and simultaneous, pitch spectral contents are present at twice this frequency. This condition is defined as a hypothesis testing problem using the following formulation where $\mathcal{H}_{0}$ means the resonance condition is not met and $\mathcal{H}_{1}$ means the resonance condition is met,

$$
\begin{array}{ll}
\mathcal{H}_{0}: \mathcal{P}_{\phi^{2} \theta}(\omega) \leq \overline{\mathcal{P}}, & \forall \omega \in \mathbb{R} \\
\mathcal{H}_{1}: \mathcal{P}_{\phi^{2} \theta}(\omega)>\overline{\mathcal{P}}, & \forall \omega \in \mathbb{R}
\end{array}
$$

where $\overline{\mathcal{P}}$ is a power threshold. Instead of using directly the cross-spectrum, a spectral correlation coefficient is exploited,

$$
\mathcal{S}_{\phi^{2} \theta} \triangleq \frac{\sigma_{\phi^{2} \theta}^{2}}{\sqrt{\sigma_{\phi^{2}}^{2} \sigma_{\theta}^{2}}}
$$

where $\sigma_{\phi^{2} \theta}^{2}$ is the average power of the cross-correlation of $\phi^{2}$ and $\theta, \sigma_{\phi^{2}}^{2}$ is the average power of $\phi^{2}$, and $\sigma_{\theta}^{2}$ is the average power of $\theta$. The detection problem can then be rewritten as

$$
\begin{aligned}
& \mathcal{H}_{0}: \mathcal{S}_{\phi^{2} \theta} \leq \overline{\mathcal{S}} \\
& \mathcal{H}_{1}: \mathcal{S}_{\phi^{2} \theta}>\overline{\mathcal{S}}
\end{aligned}
$$

where $\overline{\mathcal{S}}$ is a measure of the level of spectral correlation.

Variance will later be needed in a region of the spectral range for purposes of robustification and is readily available from the already calculated spectra as

$$
\sigma_{x y}^{2}\left(\omega_{1}, \omega_{2}\right)=\frac{1}{2 \pi} \int_{\omega_{1}}^{\omega_{2}} \mathcal{P}_{x y}(\omega) \mathrm{d} \omega
$$

\section{Phase Condition - Generalized Likelihood Ratio Test}

The phase correlation condition that shows the presence of resonance is expressed through the correlation between $\phi^{2}$ and $\theta$. A statistical test to show whether there is significant correlation between roll squared and pitch is therefore to test whether the product $\phi^{2}(t) \theta(t)$ belongs to the distribution $\mathcal{H}_{0}$ where no resonance is present, or the distribution deviates from normal and belongs to a $\mathcal{H}_{1}$ distribution where parametric resonance is present. To perform such test and ensure the false alarm probability is kept very low, a test statistics to be used for change detection is first derived. One challenge is that the parameters for the $\mathcal{H}_{1}$ distribution are not known. All that is known is that the distribution must deviate from that observed under $\mathcal{H}_{0}$ and the change must be significant enough to trigger false alarms with a very low probability. The properties, in particular the distribution and the parameters of the test statistics, were therefore first investigated in the $\mathcal{H}_{0}$ and $\mathcal{H}_{1}$ cases. This was done above where a Weibull distribution was found to give a good fit to observations. A dedicated detector is then derived for this distribution, and since the change is unknown, a generalized likelihood ratio test (GLRT) approach is taken. This leads to a test statistics for the double Weibull GLRT test. In order to find a threshold for change detection that gives a desired false alarm probability, it is then investigated whether the GLRT test statistics fulfills theoretical requirements of independent and identically distributed (IID) samples and when this is found not to be fulfilled in practice, the actual distribution of the test statistics under $\mathcal{H}_{0}$ is investigated to determine a threshold from observations that gives a desired (low) false alarm probability.

Assume that the driving signal $d(t)$ (27) is a realization of a double Weibull random process. Then the distribution of $N$ independent and identically distributed (IID) samples of $d(t)$ is characterized by the probability density function

$$
p(\mathbf{d})=\left(\frac{\beta}{2 v^{\beta}}\right)^{N} \prod_{i=0}^{N-1}\left[\left|d_{i}\right|^{\beta-1} \exp \left(-\left(\frac{\left|d_{i}\right|}{v}\right)^{\beta}\right)\right]
$$

where the parameters are referred to as the vector $\boldsymbol{\theta}=[v, \beta]^{T}$ in the sequel and $\mathbf{d}=\left[d_{0}, d_{1}, \ldots, d_{N-1}\right]^{T}$.

The detection of parametric roll can be formulated as a parameter test of the probability density function

$$
\begin{aligned}
& \mathcal{H}_{0}: \boldsymbol{\theta}=\boldsymbol{\theta}_{0} \\
& \mathcal{H}_{1}: \boldsymbol{\theta}=\boldsymbol{\theta}_{1}
\end{aligned}
$$

where $\boldsymbol{\theta}_{0}$ is known and it represents $\mathcal{W}_{2}$ in the non-resonant case, whereas $\boldsymbol{\theta}_{1}$ is unknown and it describes the parametric resonant case. By applying the generalized likelihood ratio test, the detector decides $\mathcal{H}_{1}$ if

$$
L_{G}(\mathbf{d})=\frac{p\left(\mathbf{d} ; \hat{\boldsymbol{\theta}}_{1}, \mathcal{H}_{1}\right)}{p\left(\mathbf{d} ; \boldsymbol{\theta}_{0}, \mathcal{H}_{0}\right)}>\gamma
$$

where the unknown parameter vector $\theta_{1}$ is replaced with its maximum likelihood estimate (MLE) $\hat{\boldsymbol{\theta}}_{1}$, and $\gamma$ is the threshold given by the desired probability of false alarms.

The first step in computing $L_{G}$ is to determine $\hat{\boldsymbol{\theta}}_{1}=$ $\left[\hat{v}_{1}, \hat{\beta}_{1}\right]^{T}$, therefore we need to maximize $p\left(\mathbf{d} ; \hat{\boldsymbol{\theta}}_{1}, \mathcal{H}_{1}\right)$. Given $p\left(\mathbf{d} ; \hat{\boldsymbol{\theta}}_{1}, \mathcal{H}_{1}\right)$ the estimates of the parameters $v_{1}$ and $\beta_{1}$ are computed as

$$
\frac{\partial \ln p\left(\mathbf{d} ; \hat{\boldsymbol{\theta}}_{1}, \mathcal{H}_{1}\right)}{\partial \theta_{j}}=0
$$

which results in

$$
\begin{aligned}
& \hat{v}_{1}=\left(\frac{1}{N} \sum_{i=0}^{N-1}\left|d_{i}\right|^{\hat{\beta}_{1}}\right)^{\frac{1}{\hat{\beta}_{1}}} \\
& \frac{1}{\hat{\beta}_{1}}=\frac{\sum_{i=0}^{N-1}\left|d_{i}\right|^{\hat{\beta}_{1}} \ln \left|d_{i}\right|}{\sum_{i=0}^{N-1}\left|d_{i}\right|^{\hat{\beta}_{1}}}-\frac{1}{N} \sum_{i=0}^{N-1} \ln \left|d_{i}\right| .
\end{aligned}
$$


Balakrishnan and Kateri [37] have shown that $\hat{\beta}_{1}$ exists, it is unique, and its value is given by the intersection of the curve $1 / \hat{\beta}_{1}$ with the right-hand side of (38).

Having determined the MLEs $\hat{v}_{1}$ and $\hat{\beta}_{1}$ it is then possible to derive an explicit form for the detector. By taking the natural logarithm of both sides of (36),

$$
\begin{aligned}
& \left(\frac{\beta_{1}}{2 v_{1}^{\beta_{1}}}\right)^{N} \prod_{i=0}^{N-1}\left[\left|d_{i}\right|^{\beta_{1}-1} \exp \left(-\left(\frac{\left|d_{i}\right|}{v_{1}}\right)^{\beta_{1}}\right)\right]
\end{aligned}
$$

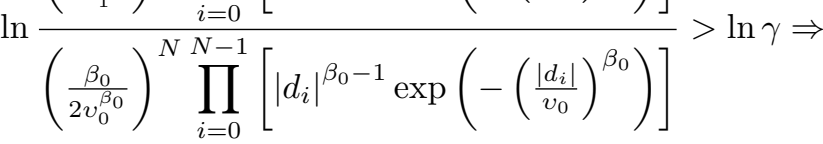

$$
\begin{aligned}
& N \ln \left(\frac{\beta_{1}}{\beta_{0}} \frac{v_{0}^{\beta_{0}}}{v_{1}^{\beta_{1}}}\right)+\left(\beta_{1}-\beta_{0}\right) \sum_{i=0}^{N-1} \ln \left|d_{i}\right|-\sum_{i=0}^{N-1}\left(\frac{\left|d_{i}\right|}{v_{1}}\right)^{\beta_{1}} \\
& +\sum_{i=0}^{N-1}\left(\frac{\left|d_{i}\right|}{v_{0}}\right)^{\beta_{0}}>\gamma^{\prime}
\end{aligned}
$$

where the parameters $\left[\beta_{1}, v_{1}\right]$ are replaced by their estimates, and $\gamma^{\prime}=\ln \gamma$.

If the shape parameter is the same under both hypothesis, $\beta_{1}=\beta_{0}=\beta$, then from (39),

$$
N \beta \ln \left(\frac{v_{0}}{v_{1}}\right)+\frac{v_{1}^{\beta}-v_{0}^{\beta}}{\left(v_{0} v_{1}\right)^{\beta}} \sum_{i=0}^{N-1}\left|d_{i}\right|^{\beta}>\gamma^{\prime}
$$

and inserting (37), the GLRT simplifies to,

$$
N \beta \ln \left(\frac{v_{0}}{v_{1}}\right)+N \frac{v_{1}^{\beta}-v_{0}^{\beta}}{v_{0}^{\beta}}>\gamma^{\prime} .
$$

Hence for the time window $k$ the test statistics $g(k)$ is

$$
g(k)=N \beta \ln \left(\frac{v_{0}}{v_{1}}\right)+N \frac{v_{1}^{\beta}-v_{0}^{\beta}}{v_{0}^{\beta}} .
$$

1) Theoretical Threshold: According to the NeymanPearson theorem [38] given the signal $d(t)$, which behaves according to the PDF $p\left(d ; \mathcal{H}_{0}\right)$ under the hypothesis $\mathcal{H}_{0}$, the threshold $\gamma$ that maximizes the probability of detection $P_{D}$ is found from

$$
P_{F A}=\int_{\left\{d: L_{G}(d)>\gamma\right\}} p\left(d ; \mathcal{H}_{0}\right) \mathrm{d} d,
$$

where $P_{F A}$ is the desired probability of false alarm.

For large data records $(N \rightarrow \infty)$, an asymptotic result exists for the modified GLRT statistic $2 \ln L_{G}$ (d), but in the present case, records are short and asymptotic results fall short.

\section{Insensitivity to Forced Roll}

For a real ship sailing in oblique short-crested seaways some forced roll with frequency equal to the encounter frequency will always occur. This does not obscure the proposed detection schemes since both the spectral correlation coefficient and the GLRT for non-Gaussian processes are insensitive to forced roll. Mathematical proofs are provided in Appendix B.
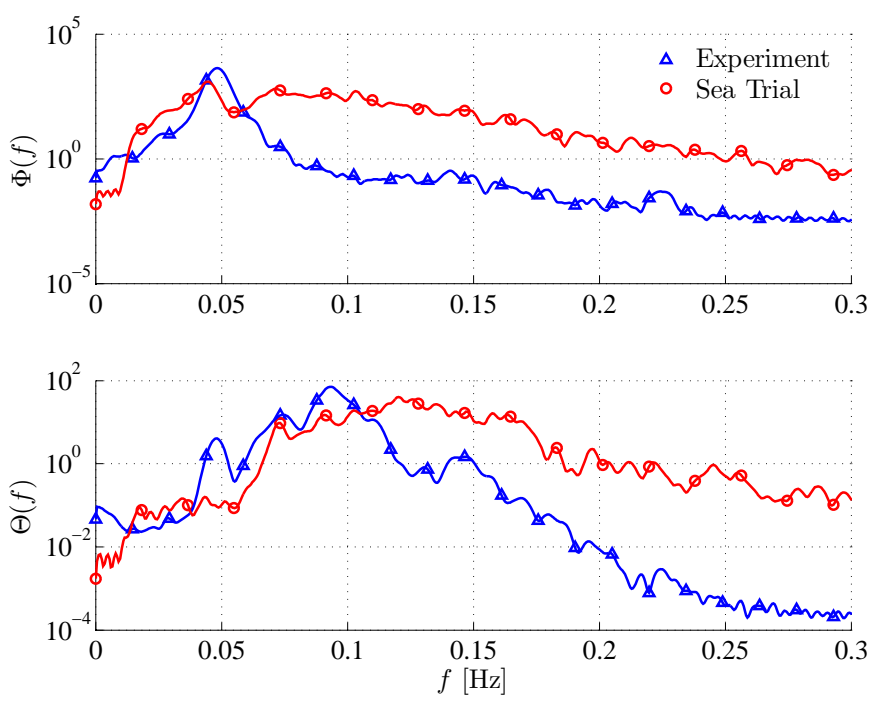

Fig. 7. Comparison of power spectra for experimental and sea trial time series.

\section{MONITORING SYSTEM ENGINEERING}

The proposed detection schemes rely on assumptions, which in general may not be completely fulfilled during real navigation operations. Issues as wide-band roll and pitch signals; unavailability of prior information to generate the hypothesis $\mathcal{H}_{0}$; high correlation of the driving signal must be carefully addressed to obtain a robust and reliable monitoring system.

\section{A. The Spectral Correlation Detector}

The spectral correlation performs best when the signals at hand have a narrow band power spectrum because in that case the Fourier transform of the convolution between the first harmonic of roll squared with the first harmonic of pitch will be zero most of time except when parametric roll is developing. However in real sailing conditions the wave spectrum exciting the ship motions can be rather broad, and it induces ship responses whose frequency content spans over a wide range of frequencies as well. Fig. 7 compares the power spectra of time series recorded during an experiment in a towing tank, and during real navigation across the Atlantic.

It is evident that during real navigation the roll and pitch motions have an energy content larger than $10^{-1}$ over a wide range of frequencies, and this will contribute to determine a significant spectral correlation also in these regions of frequencies where parametric roll is not likely. Consequently this may determine an increase in the number of false alarms issued by the detector; hence the necessity of robustifying the spectral correlation method.

The robustification relies on the use of bandpass filters in order to reduce the roll and pitch motion to be narrow band signals. The pass-bands of the filters are centered about $\omega_{\phi}$ and $\omega_{e}=2 \omega_{\phi}$, to select the frequency ranges where parametric roll resonance can develop. Therefore the spectral correlation coefficient takes the form

$$
{ }^{f} \mathcal{S}_{\phi^{2} \theta}=\frac{{ }^{f} \sigma_{\phi^{2} \theta}^{2}}{\sqrt{\sigma_{\phi^{2}}^{2} \sigma_{\theta}^{2}}}
$$




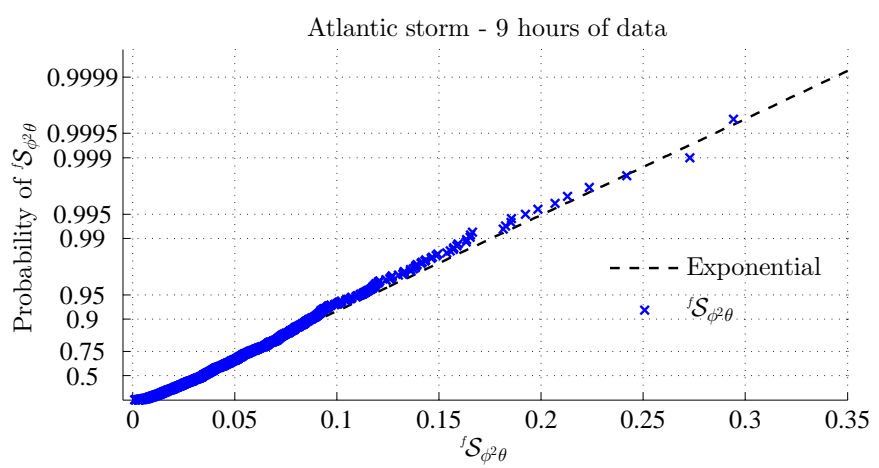

Fig. 8. Probability plot of the spectral correlation ${ }^{f} \mathcal{S}_{\phi^{2} \theta}$ computed on data recorded in heavy weather with forced roll. An exponential distribution fits the data very well. Data from the Atlantic passage of Clara Maersk.

where the superscript $f$ addresses that in the computation of the cross-spectrum the filtered signals are utilized. Note that the normalization factor in (44) is still calculated by means of the raw roll and pitch signals; this is because the spectral correlation index provides a measure of the relative energy contribution of parametric resonance to the total energy driving the roll motion.

1) Threshold Selection: The properties of the statistical test are illustrated in Fig. 8 where the spectral correlation $\mathcal{S}_{\phi^{2} \theta}$ is calculated every $22 \mathrm{~s}$ on overlapping windows that are four times longer. Fig. 8 shows the probability plot of the spectral correlation and an approximating exponential distribution,

$$
p\left({ }^{f} \mathcal{S}_{\phi^{2} \theta} ; \mathcal{H}_{0}\right)=\frac{1}{\mu_{S}} \exp \left(-\frac{{ }^{f} \mathcal{S}_{\phi^{2} \theta}}{\mu_{S}}\right)
$$

that was found to provide a good fit to data.

The data used are recordings from the $33000 \mathrm{~m}^{3}$ displacement container vessel Clara Maersk during 9 hours of navigation through an Atlantic storm. Roll resonance was not believed to be present so these data represent the $\mathcal{H}_{0}$ condition. A threshold can then be readily chosen to obtain a desired probability of false alarms. A false alarm probability of $P_{F A}=0.01$ is obtained for threshold 0.18 , and $P_{F A}=0.001$ for 0.26 .

\section{B. The $\mathcal{W}_{2}$-GLRT detector}

The $\mathcal{W}_{2}$-GLRT detector (39) was derived assuming that the $p\left(\mathbf{d} ; \boldsymbol{\theta}_{0}, \mathcal{H}_{0}\right)$ is known a priori. Actually this is not the case, and an estimate of $\boldsymbol{\theta}_{0}$ is to be computed. Since the sea state varies over time and $\boldsymbol{\theta}_{0}$ describes non sub-harmonic roll motion induced by the wave motion, the hypothesis $\mathcal{H}_{0}$ needs to be constantly updated to track changes in sea state. This implicitly provides robustness to the $\mathcal{W}_{2}$-GLRT detector: by changing $\mathcal{H}_{0}$ with a suitable estimate of $\boldsymbol{\theta}_{0}$, the detector becomes insensitive to the current sea state.

Assuming to be at time $t=T$ two phases can be distinguished:

- Estimation phase: $\hat{\boldsymbol{\theta}}_{0}$ is computed via MLE (37)-(38) on data logged within the time window $\left[T-M_{\text {det }}-M_{\text {est }}+\right.$ $\left.1, T-M_{\mathrm{det}}\right]$

- Detection phase: $\hat{\boldsymbol{\theta}}_{1}$ is computed via MLE (37)-(38) on data logged within the time window $\left[T-M_{\text {det }}+1, T\right]$.
Hence the test statistics (39) (or (42)) is calculated and compared against the threshold

where $M_{\text {est }}$ is the estimation window, and $M_{\text {det }}$ is the detection window.

When the $\mathcal{W}_{2}$-GLRT detector is started an initialization phase is run, where the first $M_{\text {est }}$ data samples are used to generate the initial $\hat{\boldsymbol{\theta}}_{0}$. During this time the detection is idle since the hypothesis $\mathcal{H}_{0}$ is not available yet.

The $\mathcal{W}_{2}$-GLRT detector further assumes that the double Weibull PDFs for the non-resonant and resonant case differ both for the shape parameter $\beta$ and for the scale parameter $v$. To computationally achieve a precise estimate of $\beta$ requires to solve a nonlinear equation within each iteration of the GLRT algorithm, as shown in Section III-C. This obviously determines an undesired computational burden and time consumption. However Fig. 6 shows that the double Weibull PDFs for the non-resonant and resonant case differ mainly for the scale parameter $\left(v_{\mathrm{r}} \approx 31 v_{\mathrm{nr}}\right)$ whereas the shape parameter deviates for less than $2 \%$. Therefore it is assumed that the shape parameter is constant - i.e. $\beta_{0}=\beta_{1}=\beta$ - and that the $\mathcal{W}_{2}$-GLRT detector only looks for variations in the scale parameter $v$.

1) Threshold Selection: The derivation of the double Weibull GLRT detector was based on an IID assumption. Fig. 9 shows the autocorrelation $\mathcal{R}_{d d}$ for $d(k)$, from which it is evident that the driving signal is neither independently distributed nor white. Further, the assumption on identical distribution implies stationarity, and it is known that it is satisfied only to a certain extent. Wave elevation for a fully developed sea, and in turn ship motions, can be considered quasi-stationary for a period of time that depends on weather conditions [39].

If the derived detection scheme is applied disregarding the IID assumption

- the detector will be suboptimal

- the false alarm rate will be higher than expected if the threshold was chosen according to Section III-C1

- the change in statistic over time will affect the performance of the detector.

In this paper, the last item is in practice handled by using data windows, which are sufficiently small to approximate stationarity.

The correlation structure, which is clearly seen in Fig. 9, should ideally be included in (36). However, this is a very hard task in connection to the double Weibull distribution. Another, and maybe more important, issue is the relation between the lack of stationarity and the large horizon needed to estimate the correlation structure.

For these reasons, the approach taken in this paper is to apply the detection scheme derived under the IID assumption but find the threshold from the cumulative density function of the test statistic under non-resonant conditions. In practice, non-resonant conditions can be determined using a sliding window over immediately past data where both the frequency and the synchronization detectors indicated a $\mathcal{H}_{0}$ condition.

In rigorous terms, the threshold $\gamma_{g}$ to obtain a sufficiently low false alarm rate, depends on the statistics of $g(k)$ in (42) 


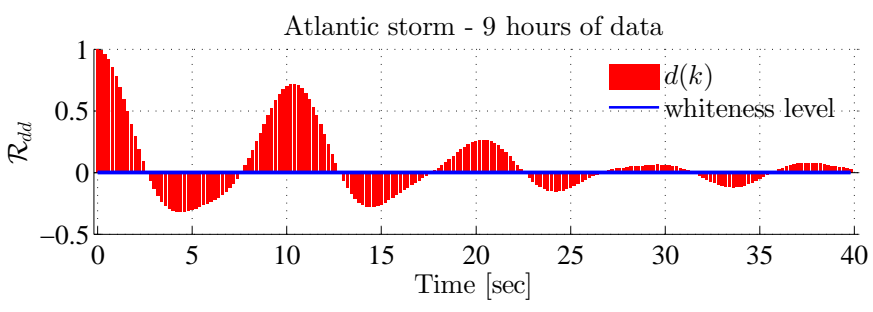

Fig. 9. Plot of autocorrelation $\mathcal{R}_{d d}$ for the driving signal $d(k)$ shows that samples are heavily correlated due to the narrow-band nature of motions.

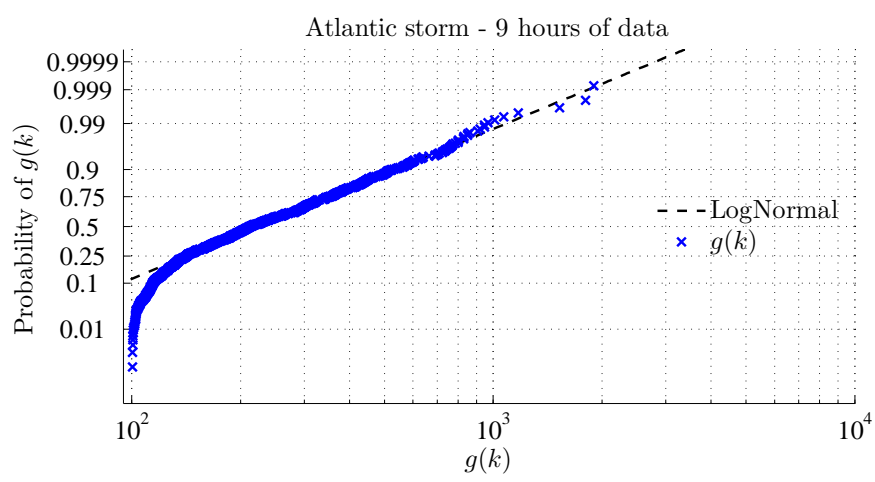

Fig. 10. GLRT test statistics $g(k)$ fitted against a Lognormal distribution. Data from Atlantic passage of Clara Maersk during a storm.

under hypothesis $\mathcal{H}_{0}$. Given that $g(k)$ behaves according to a PDF $p\left(g ; \mathcal{H}_{0}\right)$, which is estimated from data, the threshold is selected from

$$
P_{F A}=\int_{\left\{g: g>\gamma_{g}\right\}} p\left(g ; \mathcal{H}_{0}\right) \mathrm{d} g .
$$

Estimation of the density function $p\left(g ; \mathcal{H}_{0}\right)$ from data is illustrated in Fig. 10. A Lognormal distribution with the PDF,

$$
p\left(g ; \mathcal{H}_{0}\right)=\frac{1}{\sqrt{2 \pi} \sigma_{0 g} g} \exp \left(-\frac{\left(\ln (g)-\mu_{0 g}\right)^{2}}{2 \sigma_{0 g}^{2}}\right)
$$

fits the upper part (tail) of the distribution well. The tail of the distribution are particularly important to determine $P_{F A}$, the $g(k)$ and data in the plot were censored to the range $g(k)>$ 100. Having obtained the estimated parameters $\mu_{0 g}$ and $\sigma_{0 g}$ of the Lognormal distribution for $g(k)$ under $\mathcal{H}_{0}$, the threshold for a desired false alarm probability is obtained from (46) with the distribution from (47),

$$
1-P_{F A}=\Phi\left(\frac{\ln \left(\gamma_{g}\right)-\mu_{0 g}}{\sigma_{0 g}}\right)
$$

where $\Phi(x)=\frac{1}{2}\left(1+\operatorname{erf}\left(\frac{x}{\sqrt{2}}\right)\right)$ is the standard (zero mean, unit variance) Gaussian distribution function. Hence,

$$
\gamma_{g}=\exp \left(\mu_{0 g}+\Phi^{-1}\left(1-P_{F A}\right) \sigma_{0 g}\right)
$$

For the hypothesis $\mathcal{H}_{0}$ shown in Fig. 10 the Lognormal fit gives a threshold $\gamma_{g}=1080$ for $P_{F A}=0.01$ and $\gamma_{g}=1870$ for $P_{F A}=0.001$.

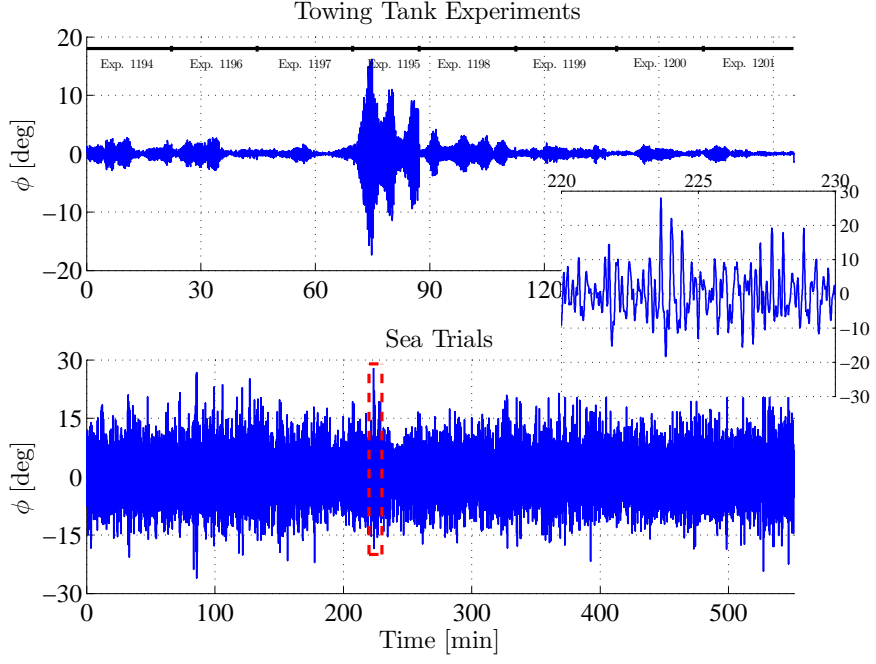

Fig. 11. Top: roll motion time series recorded from experimental runs. Experiment 1195 is the only one where parametric roll clearly developed. Bottom: roll motion time series recorded during navigation across the North Atlantic Ocean.

\section{Implementation}

The results shown in Section $\mathrm{V}$ were obtained using the estimation window $M_{\text {est }} \approx 30 \mathrm{~min}$, the detection window equivalent to four roll periods, $M_{\mathrm{det}}=4 \cdot T_{\phi}$, where $T_{\phi}$ is the roll natural period, and $75 \%$ window overlap. Both the spectral correlation detector and the double Weibull GLRT use these settings.

\section{DETECTION SCHEMES VALIDATION}

The performance of the proposed detection schemes for parametric roll have been validated on two data sets. In order to simulate a continuous navigation the single records of each data set have been stitched together. A smoothing filter was applied around the stitching points to avoid that sudden fictitious variations within the signals at hand could trigger an alarm. Hence the roll time series scrutinized are those shown in Fig. 11.

\section{A. Model Tank Data}

The first data set (Fig. 11, top plot) consists of eight experiments run in irregular waves scenario $^{1}$ at the Marine Technology Centre in Trondheim. The vessel used for the tank experiments is a 1:45 scale model of a $76.000 \mathrm{~m}^{3}$ displacement, $281 \mathrm{~m}$ long container ship. The roll period is $21 \mathrm{~s}\left(\omega_{\phi}=0.298 \mathrm{rad} / \mathrm{s}\right)$. The detailed model and all the hydrodynamic coefficients can be found in [31].

Table I summarizes the parameters of the experimental runs, where $T_{\exp }$ is the duration of the experiment, $H_{s}$ is the significant wave height, $T_{p}$ is the wave peak period, $\phi_{\max }$ is the maximum roll angle achieved during the experiment, and $\sigma_{\phi}$ is the standard deviation of the roll motion. All data in Table I are in full scale.

${ }^{1}$ The terminology irregular waves means that the wave motion used to excite the vessel is generated by the superposition of multiple sinusoidal waves centered at different frequencies, and described by a given power spectrum. In regular waves the vessel is excited by a single sinusoidal wave. 
TABLE I

IRREGULAR WAVE EXPERIMENTS

\begin{tabular}{rrrrrr}
\hline Exp. & $T_{\exp }[\mathrm{s}]$ & $H_{s}[\mathrm{~m}]$ & $T_{p}[\mathrm{~s}]$ & $\left|\phi_{\max }\right|\left[^{\circ}\right]$ & $\sigma_{\phi}\left[^{\circ}\right]$ \\
\hline 1194 & 1338 & 9 & 13.54 & 2.91 & 1.02 \\
1195 & 1048 & 9 & 13.54 & 17.24 & 5.53 \\
1196 & 1345 & 9 & 13.54 & 2.88 & 0.97 \\
1197 & 1504 & 7 & 13.54 & 1.68 & 0.49 \\
1198 & 1522 & 7 & 13.54 & 4.23 & 1.31 \\
1199 & 1582 & 9 & 13.54 & 1.83 & 0.55 \\
1200 & 1369 & 9 & 13.54 & 1.74 & 0.46 \\
1201 & 1419 & 9 & 13.54 & 1.45 & 0.41 \\
\hline
\end{tabular}

TABLE II

SEA TRIALS

\begin{tabular}{rrrr}
\hline $\mathrm{ST}$ & $H_{s}[\mathrm{~m}]$ & $T_{e}[\mathrm{~s}]$ & \multicolumn{1}{c}{$\chi_{e}\left[^{\circ}\right]$} \\
\hline 82 & $5-8$ & $5-6$ & 150 \\
83 & $5-8$ & $5-6$ & 150 \\
84 & $8-10$ & $5-8$ & 150 \\
85 & $10-12$ & $7-10$ & 150 \\
86 & $10-12$ & - & $135-150$ \\
87 & $10-11$ & - & 160 \\
88 & $8-11$ & 6 & 150 \\
89 & $8-10$ & $5-7$ & $150-170$ \\
90 & $8-10$ & - & 170 \\
91 & $8-10$ & $5-6$ & 150 \\
92 & 11 & 11 & - \\
\hline
\end{tabular}

It is emphasized that, although the vessel experienced parametric roll only once, all the tank tests were made to trigger the phenomenon, but in the irregular wave scenario it is difficult to obtain a fully developed parametric roll resonance as consecutive wave trains may not fulfill all the conditions for its existence. The experimental data set is used to evaluate the capability of the detectors to timely catch the onset of parametric roll.

\section{B. Atlantic Passage Data}

The second data set (Fig. 11, bottom plot) consists of full scale data recorded on board the container ship Clara Maersk crossing the North Atlantic Ocean. The length of the vessel was $L_{\mathrm{PP}}=197 \mathrm{~m}$, and its displacement was $\nabla=33000 \mathrm{~m}^{3}$; its roll period was $22 \mathrm{~s}\left(\omega_{\phi}=0.286 \mathrm{rad} / \mathrm{s}\right)$ in the voyage condition.

Time series data used here correspond to nine hours of navigation. Table II summarizes the sailing conditions, where $H_{s}$ is the significant wave height, $T_{e}$ is the encounter wave period, and $\chi_{e}$ is the wave encounter angle. Values have been manually observed and are subject to uncertainty.

For this data set there was no prior awareness about the onset of parametric roll resonance; hence the assessment of detections and/or false alarms was done by visual inspection of the time series around the alarm time. The real navigation data set is used to ensure the insensitivity to usual forced roll.

\section{Spectral Correlation Detector Validation}

Figs. 12-13 show the outcome of the spectral correlation detector versus its robustified version after processing the model and full scale data sets.

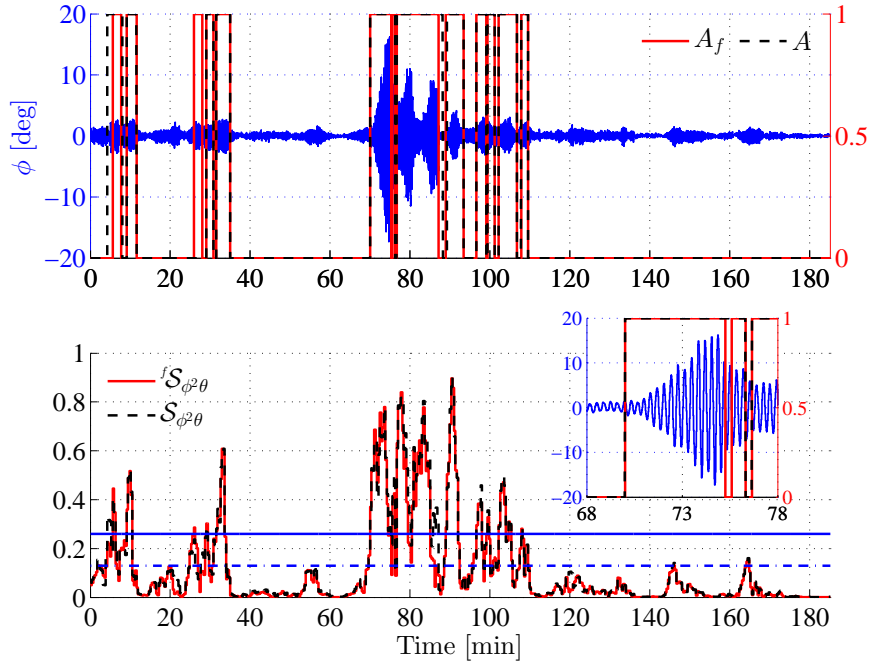

Fig. 12. Normal and robust spectral correlation detector on the experimental data set.

On the experimental data set the spectral correlation and the robust spectral correlation detectors show similar good performance. Fig. 12 illustrates that the major parametric roll event that occurs between $t=70 \mathrm{~min}$ and $t=90 \mathrm{~min}$ is promptly detected when the roll angle is about $3^{\circ}$ by both detectors. The other alarms issued by the detectors within the first 40 minutes and after 100 minutes are currently considered false alarms since there is no visual evidence that parametric roll is taking place. However it must be noted that in all experiments the wave spectrum wan tuned to induce parametric resonance, that is to fulfill the frequency condition $\omega_{e} \approx 2 \omega_{\phi}$. Therefore, although parametric roll did not develop to its dangerous level, its sparkle was present and that is what triggered these alarms. The difference between the normal and robustified spectral correlation detector is very marginal, and this is due to the very narrow band wave spectrum used to excite the model.

Fig. 13 clearly acknowledge the importance of pre-filtering the signals for the spectral correlation index. Without the pre-filtering, the wide spectra of pitch and roll in the sea trial data give many false alarms, which are reduced to three when the robustified detector is used. Pre-filtering hence ensures robustness, although few alarms are still issued by the detector. By looking at the overall performance over 9 hours of heavy roll motion it can be concluded that insensitivity to synchronous roll is achieved.

\section{Phase Synchronization Detector Validation}

Fig. 14 shows the detection performance of the double Weibull GLRT detector of phase synchronization on the experimental data set. The parametric roll event is promptly detected when the roll angle is smaller than $3^{\circ}$, and the alarm lasts for the entire duration of the major event.

Fig. 15 shows the performance of the $\mathcal{W}_{2}$-GLRT on the full scale data set. The double Weibull GLRT detector issues one alarm, which by visual investigation is classified as false alarm. Although the $\mathcal{W}_{2}$-GLRT is not false alarm free, its overall 


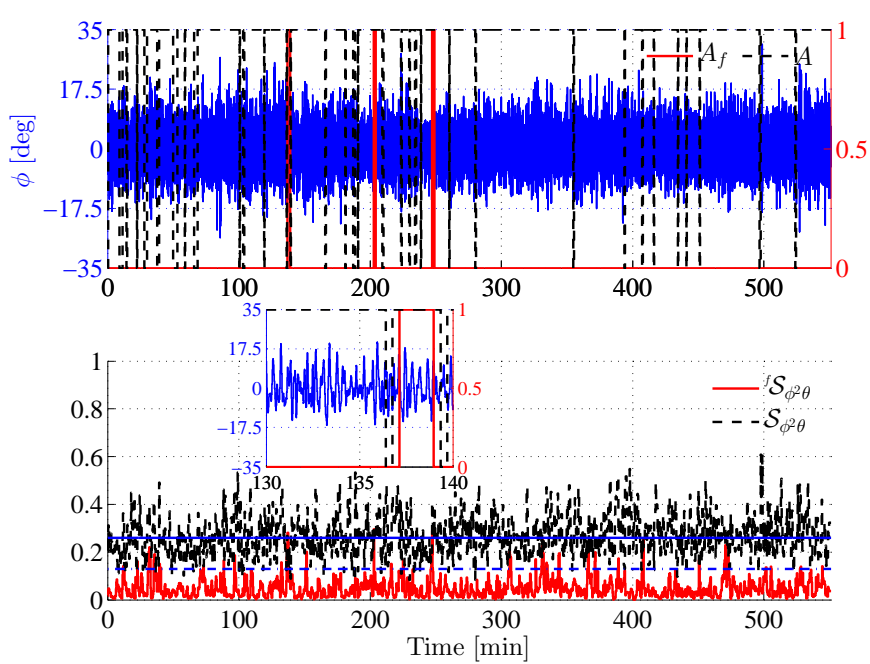

Fig. 13. Spectral correlation detector on the sea trial data set with broad spectral content in pitch and roll: a large amount of false alarms with the unfiltered signals are avoided by applying the pre-filtering.
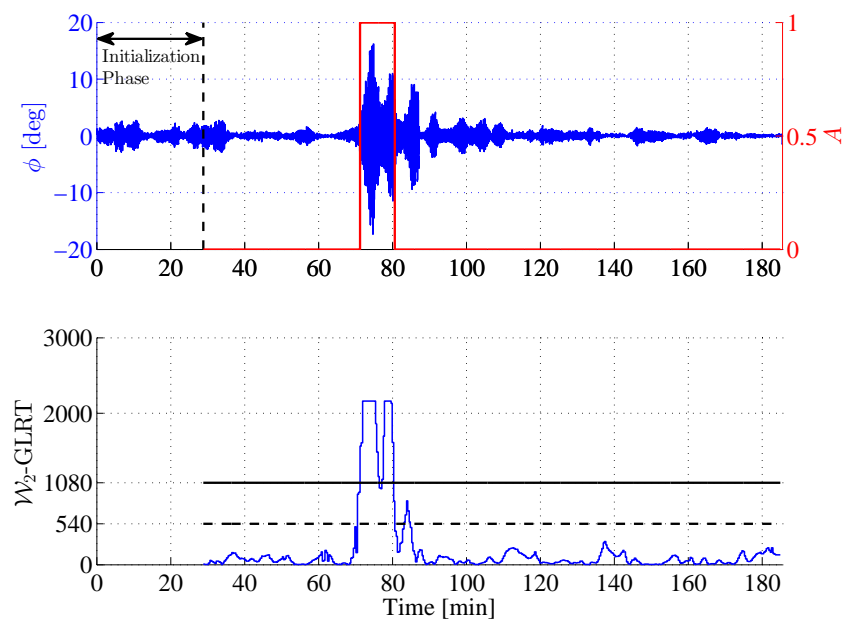

Fig. 14. $\mathcal{W}_{2}$-GLRT on the experimental data set.

behavior on this data set supports the choice of the double Weibull distribution for describing the statistical properties of the driving signal under the hypothesis $\mathcal{H}_{0}$.

\section{E. Robust Performance by Combined Hypothesis Testing}

The spectral correlation detector and the double Weibull GLRT detector each shown good performance providing a timely detection of the parametric roll event that occurs within the experimental time series, giving a moderate number of false alarms when applied to the experimental and the real navigation data sets. However, it is only the combination of the detectors that can provide a $P_{F A}$ rate that is acceptable. With update interval $T_{\phi} \approx 22 \mathrm{~s}$, a one-month interval between false alarms is equivalent to $P_{F A} \simeq 10^{-5}$ for tests with independent samples. Combining the two detectors provide the false alarm probability,

$P_{F A}\left(g,{ }^{f} \mathcal{S}_{\phi^{2} \theta}\right) \triangleq P_{F A}\left(g>\gamma_{g} \cap{ }^{f} \mathcal{S}_{\phi^{2} \theta}>\overline{\mathcal{S}}\right)$
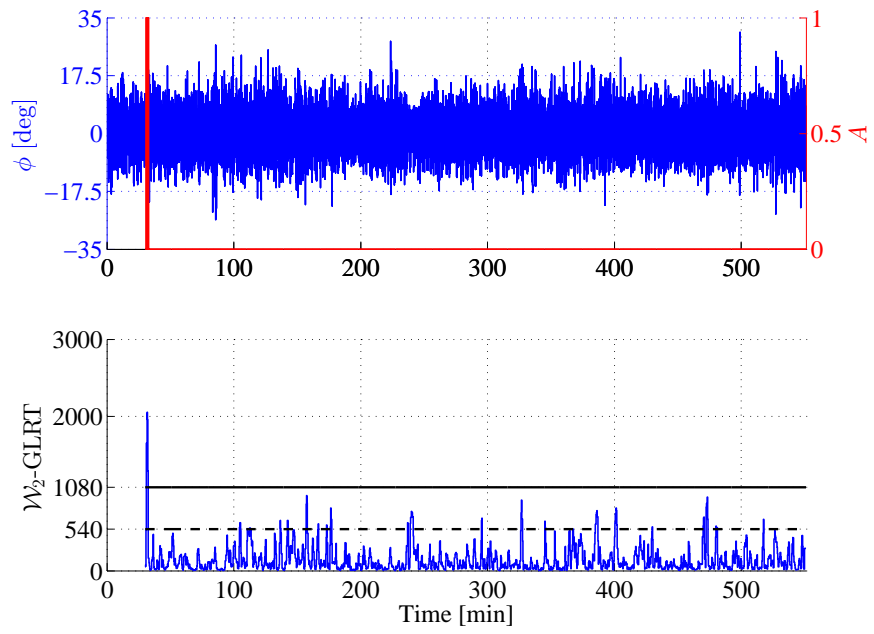

Fig. 15. $\mathcal{W}_{2}$-GLRT on the sea trial data set.
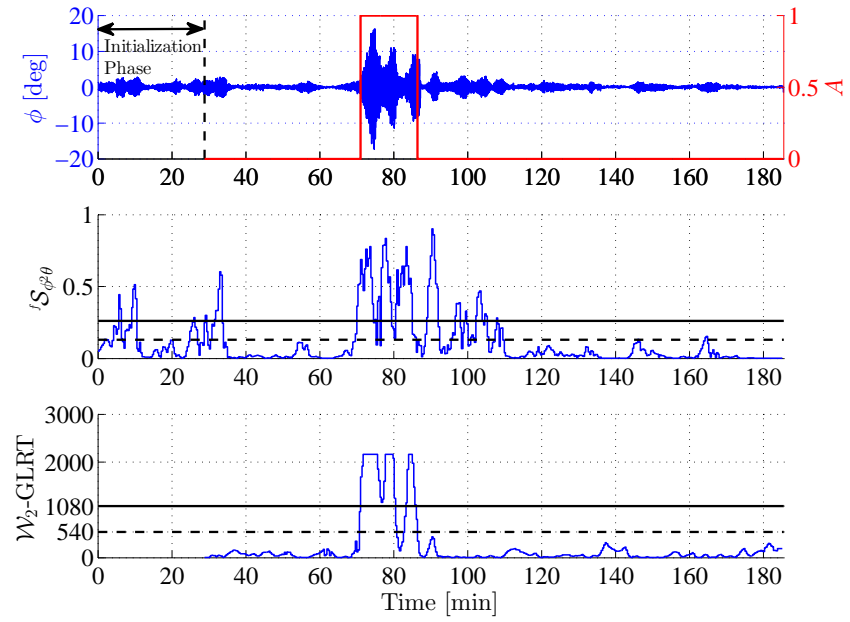

Fig. 16. Performance of the monitoring system on the experimental data set.

$$
=P_{F A}\left(g>\left.\gamma_{g}\right|^{f} \mathcal{S}_{\phi^{2} \theta}>\overline{\mathcal{S}}\right) P_{F A}\left({ }^{f} \mathcal{S}_{\phi^{2} \theta}>\overline{\mathcal{S}}\right) .
$$

The effect of combined hypothesis testing is a drastically reduced false alarm probability. If $P_{F A}\left(g>\gamma_{g} \mid{ }^{f} \mathcal{S}_{\phi^{2} \theta}>\overline{\mathcal{S}}\right) \simeq P_{F A}\left(g>\gamma_{g}\right), P_{F A}\left(g,{ }^{f} \mathcal{S}_{\phi^{2} \theta}\right) \simeq$ $P_{F A}\left(g>\gamma_{g}\right) P_{F A}\left({ }^{f} \mathcal{S}_{\phi^{2} \theta}>\overline{\mathcal{S}}\right)$, but data are not available to make this conclusion at a rigorous level. At a heuristic level, however, a vast improvement of the use of the combined detectors is illustrated in Fig. 16 for the experimental data set, and in Fig. 17 for the real navigation data.

Selecting thresholds for the combined phase and frequency condition detectors ideally should require consideration of detection probability as well,

$$
P_{D}\left(g,{ }^{f} \mathcal{S}_{\phi^{2} \theta}\right)=\int_{\mathcal{I}} p\left(g,{ }^{f} \mathcal{S}_{\phi^{2} \theta} ; \mathcal{H}_{1}\right) \mathrm{d} g \mathrm{~d}^{f} \mathcal{S}_{\phi^{2} \theta},
$$

where $\mathcal{I}=\left\{g: g \geq \gamma_{g}\right\} \cap\left\{{ }^{f} \mathcal{S}_{\phi^{2} \theta}:{ }^{f} \mathcal{S}_{\phi^{2} \theta} \geq \overline{\mathcal{S}}\right\}$. This computation would need the joint distributions under both nonresonant and resonant behaviors but sufficient data were not available and thresholds had to be selected based on $P_{F A}$ from the observed distributions. 

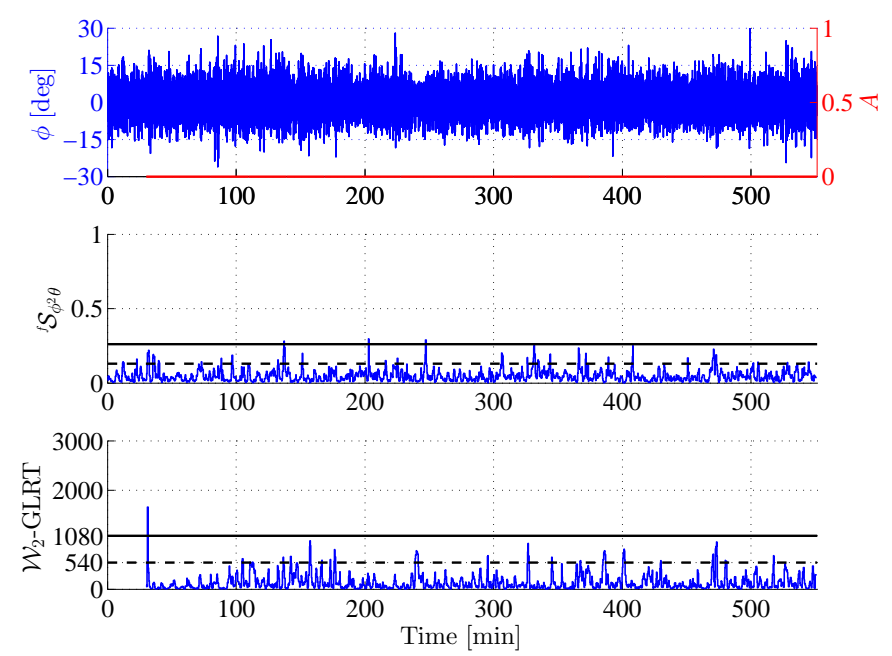

Fig. 17. Performance of the monitoring system on the real navigational data.

\section{CONCLUSIONS}

Two detection methods were proposed for the diagnosis of parametric roll resonance and were validated against model scale and full scale data.

A spectral correlation detector was developed in the frequency domain, providing an indicator for energy flowing from the pitch motion, directly excited by the waves, into roll motion causing a sub-harmonic resonance. A double Weibull GLRT detector worked in the time domain, and monitored the behavior of a driving signal carrying information about the phase correlation between roll and pitch. Robustness against usual forced roll motion was proven.

The two detectors showed very satisfactory capabilities to timely detect the onset of parametric roll, while achieving a low false alarm rate. A significant overall performance enhancement was obtained by using the two detectors for combined hypothesis testing.

The methodologies reported in this paper were included in international patent applications [40].

\section{APPENDIX A \\ PHASE SYNCHRONIZATION}

Phase synchronization occurs between roll and pitch during parametric resonance. Consider the reduced roll model

$$
\ddot{\phi}+\nu_{2} \dot{\phi}+\left(\omega_{\phi}^{2}+\kappa_{1} F \cos \omega_{e} t\right) \phi+\kappa_{3} \phi^{3}=0,
$$

where the nonlinearity is restrained to the cubic term of the restoring moment. By change of variable $\xi=\frac{1}{2} \omega_{e} t$ (A.1) can be rewritten as the nonlinear Mathieu equation

$$
\phi^{\prime \prime}+\nu \phi^{\prime}+(\delta+\varepsilon \cos 2 \xi) \phi+\kappa \phi^{3}=0,
$$

where $\delta=4 \omega_{2}^{2} / \omega_{e}^{2}, \varepsilon=4 \kappa_{1} F / \omega_{e}^{2}, \nu=2 \nu_{2} / \omega_{e}, \kappa=4 \kappa_{3} / \omega_{e}^{2}$, and the prime addresses differentiation with respect to $\xi$. When the nonlinear Mathieu equation is in the principal parametric resonance condition $(\delta \approx 1)$ the solution to first order approximation is given by

$$
\phi=\alpha(\varepsilon t) \cos \left(\frac{1}{2} \omega_{e} t-\chi(\varepsilon t)\right)+O(\varepsilon) .
$$

The amplitude $\alpha$ and phase shift $\chi$ can be determined by solving the autonomous system

$$
\begin{aligned}
\alpha^{\prime} & =-\frac{1}{4} \frac{\alpha}{\omega} \sin \chi-\nu \alpha \\
\alpha \chi^{\prime} & =2 \sigma \alpha-\frac{1}{2} \frac{\alpha}{\omega} \cos \chi-\frac{3}{2} \frac{\kappa}{\omega} \alpha^{3},
\end{aligned}
$$

where $\omega=\sqrt{\delta}=2 \omega_{\phi} / \omega_{e}$, and $\sigma$ is a detuning parameter that measures how close the system is to the principal parametric resonance condition. The steady state solution is,

$$
\begin{array}{r}
-\frac{1}{4 \omega} \sin \chi-\nu=0 \\
2 \sigma-\frac{1}{2 \omega} \cos \chi-\frac{3}{4} \frac{\kappa}{\omega} \alpha^{2}=0
\end{array}
$$

from which the amplitude and the phase shift are, to first order

$$
\begin{aligned}
\alpha & =\sqrt{\frac{8}{3} \frac{\sigma}{\kappa} \pm \frac{2}{3 \kappa} \sqrt{1-16 \nu^{2}}} \\
\tan \chi & =\frac{-4 \nu}{ \pm \sqrt{1-16 \nu^{2}}} .
\end{aligned}
$$

The phase shift depends exclusively on the system damping. At steady state it is 0 or $\pi$ according to the sign of $\cos \chi$.

\section{APPENDIX B \\ INSENSITIVITY TO FORCED ROLL}

This appendix shows that the detectors are insensitive to forced roll under a narrow-band assumption. The two detectors are treated in separate subsections.

\section{A. Frequency Condition - Spectral Correlation Detector}

Consider pitch and roll as narrow-band signals

$$
\begin{aligned}
& \theta(t) \text { s.t. } \Theta(\omega)=0 \text { for }\left|\omega-\omega_{\theta}\right| \geq \Omega_{\theta} \\
& \phi(t) \text { s.t. } \Phi(\omega)=0 \text { for }\left|\omega-\omega_{\phi}\right| \geq \Omega_{\phi}
\end{aligned}
$$

where $\Theta(\omega)$ and $\Phi(\omega)$ are the spectra of pitch and roll centered at the center frequency $\omega_{\theta}$ and $\omega_{\phi}$ respectively. The bands of the spectra are given by $B_{\theta}=\left\{\omega\right.$ s.t. $\left.\left|\omega-\omega_{\theta}\right|<\Omega_{\theta}\right\}$ and $B_{\phi}=\left\{\omega\right.$ s.t. $\left.\left|\omega-\omega_{\phi}\right|<\Omega_{\phi}\right\}$, where $W_{\theta}=2 \Omega_{\theta}$ and $W_{\phi}=$ $2 \Omega_{\phi}$ are the bandwidths. The spectral correlation coefficient $\mathcal{S}_{\phi^{2} \theta}$ is based on the computation of the cross-spectrum $P_{\phi^{2} \theta}$, the Fourier transform of the cross-correlation $r_{\phi^{2} \theta}$. Therefore,

$$
P_{\phi^{2} \theta}=\mathcal{F}\left(r_{\phi^{2} \theta}\right)=\mathcal{F}\left(\phi^{2} * \theta\right)=\overline{\mathcal{F}\left(\phi^{2}\right)} \mathcal{F}(\theta)
$$

where $\overline{\mathcal{F}\left(\phi^{2}\right)}$ is the complex conjugate.

If $\omega_{\theta}=\omega_{e}=2 \omega_{\phi}$, as in parametric resonance, then $B_{\theta}=$ $\left\{\omega\right.$ s.t. $\left.\left|\omega-2 \omega_{\phi}\right|<\Omega_{\theta}\right\}$, hence the first harmonic of the spectrum of roll squared overlaps in large part or completely the pitch spectrum. With pitch $\theta(t)$ and roll $\phi(t)$

$$
\begin{aligned}
& \theta(t)=\theta_{0}(t) \cos \left(2 \omega_{\phi} t+\psi_{\theta}(t)\right) \\
& \phi(t)=\phi_{0}(t) \cos \left(\omega_{\phi} t+\psi_{\phi}(t)\right)
\end{aligned}
$$


the spectra of pitch and the square of roll are

$$
\begin{aligned}
\Theta(\omega)= & \frac{1}{2}\left(\Theta_{i}\left(\omega-2 \omega_{\phi}\right)+\Theta_{i}\left(\omega+2 \omega_{\phi}\right)\right) \\
& -\frac{1}{2}\left(\Theta_{q}\left(\omega-2 \omega_{\phi}\right)+\Theta_{q}\left(\omega+2 \omega_{\phi}\right)\right) \\
\Phi_{2}(\omega)= & \frac{1}{2}\left(\Phi_{0}(\omega)+\Phi_{i}\left(\omega-2 \omega_{\phi}\right)+\Phi_{i}\left(\omega+2 \omega_{\phi}\right)\right) \\
& -\frac{1}{2}\left(\Phi_{q}\left(\omega-2 \omega_{\phi}\right)+\Phi_{q}\left(\omega+2 \omega_{\phi}\right)\right)
\end{aligned}
$$

where $\Theta_{i}=\mathcal{F}\left(\theta_{0}(t) \cos \left(\psi_{\theta}(t)\right)\right), \Theta_{q}=\mathcal{F}\left(\theta_{0}(t) \sin \left(\psi_{\theta}(t)\right)\right)$ are the Fourier transforms of the in-phase and quadrature components of the pitch angle, and $\Phi_{0}=\mathcal{F}\left(\phi_{0}^{2}(t)\right), \Phi_{i}=$ $\mathcal{F}\left(\phi_{0}^{2}(t) \cos \left(2 \psi_{\phi}(t)\right)\right), \quad \Phi_{q}=\mathcal{F}\left(\phi_{0}^{2}(t) \sin \left(2 \psi_{\phi}(t)\right)\right)$ are the Fourier transform of the DC, in-phase and quadrature components of the second power of roll. Thus,

$$
\begin{aligned}
P_{\phi^{2} \theta}= & \frac{1}{4}\left(\Phi_{i} \Theta_{i}\left(\omega-2 \omega_{\phi}\right)+\Phi_{i} \Theta_{i}\left(\omega+2 \omega_{\phi}\right)\right. \\
& +\Phi_{q} \Theta_{q}\left(\omega-2 \omega_{\phi}\right)+\Phi_{q} \Theta_{q}\left(\omega+2 \omega_{\phi}\right) \\
& -\Phi_{i} \Theta_{q}\left(\omega-2 \omega_{\phi}\right)-\Phi_{i} \Theta_{q}\left(\omega+2 \omega_{\phi}\right) \\
& \left.-\Phi_{q} \Theta_{i}\left(\omega-2 \omega_{\phi}\right)-\Phi_{q} \Theta_{i}\left(\omega+2 \omega_{\phi}\right)\right) .
\end{aligned}
$$

The cross-spectrum is non-zero since $\phi^{2}(t)$ and $\theta(t)$ are centered at the same frequency, hence the spectral correlation coefficient is non-zero and can be used for detection.

Consider now a ship sailing in near head seas. The lateral component of wave force excites roll motion directly, hence pitch and roll both respond at the same frequency $\omega_{e}$

$$
\begin{aligned}
& \theta=\theta_{0}(t) \cos \left(\omega_{e} t+\psi_{\theta}(t)\right) \\
& \phi=\phi_{0}(t) \cos \left(\omega_{e} t+\psi_{\phi}(t)\right) .
\end{aligned}
$$

The cross-spectrum in this case is equal to zero,

$$
\begin{aligned}
P_{\phi^{2} \theta}= & \frac{1}{4}\left[\Theta_{i}\left(\omega-\omega_{e}\right)+\Theta_{i}\left(\omega+\omega_{e}\right)\right. \\
& \left.-\Theta_{q}\left(\omega-\omega_{e}\right)-\Theta_{q}\left(\omega+\omega_{e}\right)\right] \times \\
& {\left[\Phi_{0}(\omega)+\Phi_{i}\left(\omega-2 \omega_{e}\right)+\Phi_{i}\left(\omega+2 \omega_{e}\right)\right.} \\
& \left.-\Phi_{q}\left(\omega-2 \omega_{e}\right)-\Phi_{q}\left(\omega+2 \omega_{e}\right)\right]=0
\end{aligned}
$$

since the spectra are different from zero only around $\omega=\omega_{e}$ or $\omega=2 \omega_{e}$, and they do not share frequency content. Therefore the spectral correlation coefficient is zero, and the detection is insensitive to forced roll.

\section{B. Phase Condition - $\mathcal{W}_{2}$-GLRT Detector}

Let pitch and roll be sinusoids, sampled at instants $k T_{s}$

$$
\begin{aligned}
& \theta\left(k T_{s}\right)=\theta_{0} \cos \left(\omega_{\theta} k T_{s}+\varsigma\right) \\
& \phi\left(k T_{s}\right)=\phi_{0} \cos \left(\omega_{\phi} k T_{s}\right) .
\end{aligned}
$$

With forced roll, $\left(\omega_{\theta}=\omega_{\phi}=\omega\right)$, hence

$$
\begin{aligned}
d\left(k T_{s}\right) & =\phi^{2}\left(k T_{s}\right) \theta\left(k T_{s}\right) \\
& =\phi_{0}^{2} \theta_{0} \cos ^{2}\left(\omega k T_{s}\right) \cos \left(\omega k T_{s}+\varsigma\right) .
\end{aligned}
$$

To prove that the GLRT detector (42) is not sensitive to forced roll, a constant $\Gamma$ must exist such that for any $\ln (\gamma)>\Gamma$ the detector does not trigger an alarm. In general $\Gamma$ is function of the phase shift $\varsigma$ and of the time window $N T_{s}$ used to estimate scaling and shape factors.

With $\beta_{1}=\beta_{0}$ the test statistics $g(k)$ scaled by $N$ is

$$
\begin{aligned}
\beta_{0} \ln \left(\frac{v_{0}}{\hat{v}_{1}}\right) & -\frac{1}{N} \sum_{k=0}^{N-1}\left(\frac{\left|d_{k}\right|}{\hat{v}_{1}}\right)^{\beta_{0}} \\
& +\frac{1}{N} \sum_{k=0}^{N-1}\left(\frac{\left|d_{i}\right|}{v_{0}}\right)^{\beta_{0}}>\frac{1}{N} \ln (\gamma),
\end{aligned}
$$

Under $\mathcal{H}_{0}, \hat{v}_{1}=v_{0}$, and an bound is determined from,

$$
\begin{aligned}
& \sup \left\{-\frac{1}{N} \sum_{k=0}^{N-1}\left(\frac{\left|d\left(k T_{s}\right)\right|}{\hat{v}_{1}}\right)^{\beta_{0}}\right\} \leq 0, \\
& \sup \left\{\frac{1}{N} \sum_{k=0}^{N-1}\left(\frac{\left|d\left(k T_{s}\right)\right|}{v_{0}}\right)^{\beta}\right\} \leq\left(\frac{\phi_{0}^{2} \theta_{0}}{v_{0}}\right)^{\beta_{0}} .
\end{aligned}
$$

Hence, the detector is not sensitive to forced roll when

$$
\ln (\gamma)>\Gamma \triangleq N\left(\frac{\phi_{0}^{2} \theta_{0}}{v_{0}}\right)^{\beta_{0}}
$$

This bound is time varying since $v_{0}$ and $\beta_{0}$ are changing according to weather conditions.

\section{ACKNOWLEDGEMENT}

Comments from Dr. G. Storhaug and colleagues from Det Norske Veritas (DNV) are gratefully acknowledged, and so is the inspiring collaboration with colleagues at the CeSOS center of excellence at NTNU. Data from model tests were kindly provided by Dr. T. Perez (formerly CeSOS, NTNU now Univ. of Newcastle, AUS) and Dr. G. Storhaug, DNV. Data from the Atlantic passage were logged by Prof. M. Blanke in the 1980'ties and are presented with the permission from Maersk Lines. The collaboration with T. Krarup Sørensen from Maersk and the officers of Clara Maersk are much appreciated.

\section{REFERENCES}

[1] K. L. Turner, S. A. Miller, P. G. Hartwell, N. C. MacDonald, S. H Strogatz, and S. G. Adams, "Five parametric resonances in a microelectromechanical system," Nature, vol. 396, pp. 149-152, 1998.

[2] D. J. N. Limebeer, R. S. Sharp, and S. Evangelou, "Motorcycle steering oscillations due to road profiling," Transacations of the ASME, vol. 69, pp. 724-739, 2002.

[3] W. Froude, "Remarks on Mr. Scott Russel's paper on rolling," Transactions of the Institution of Naval Architects, vol. 4, pp. 232-275, 1863.

[4] - "On the rolling of ships," Transactions of the Institution of Naval Architects, vol. 2, pp. 180-227, 1861.

[5] J. E. Kerwin, "Notes on rolling in longitudinal waves," International ShipBuilding Progress, vol. 2(16), pp. 597-614, 1955.

[6] J. R. Paulling and R. M. Rosenberg, "On unstable ship motions resulting from nonlinear coupling," Journal of Ship Research, vol. 3(1), pp. 3646, 1959.

[7] W. N. France, M. Levadou, T. W. Treakle, J. R. Paulling, R. K. Michel, and C. Moore, "An investigation of head-sea parametric rolling and its influence on container lashing systems," in SNAME Annual Meeting, 2001.

[8] K. A. Døhlie, "Parametric rolling - a problem solved?" DNV Container Ship Update, vol. 1, pp. 12-15, 2006.

[9] V. Belenky, H. Yu, and K. Weems, "Numerical procedures and practical experience of assessment of parametric roll of container carriers," in Proceedings of the 9th International Conference on Stability of Ships and Ocean Vehicles, 2006.

[10] G. Bulian, A. Francescutto, N. Umeda, and H. Hashimoto, "Qualitative and quantitative characteristics of parametric ship rolling in random waves," Ocean Engineering, vol. 35, pp. 1661-1675, 2008. 
[11] H. Hashimoto and N. Umeda, "Nonlinear analysis of parametric rolling in longitudinal and quartering seas with realistic modelling of rollrestoring moment," Journal of Marine Science and Technology, vol. 9, pp. 117-126, 2004.

[12] J. J. Jensen, "Effcient estimation of extreme non-linear roll motions using the first-order reliability method (FORM)," Journal of Marine Science and Technology, vol. 12, pp. 191-202, 2007.

[13] J. J. Jensen, P. T. Pedersen, and J. Vidic-Perunovic, "Estimation of parametric roll in stochastic seaway," in Proceedings of IUTAM Symposium on Fluid-Structure Interaction in Ocean Engineering, 2008.

[14] M. Levadou and L. Palazzi, "Assessment of operational risks of parametric roll," in SNAME Annual Meeting 2003, 2003.

[15] Y. S. Shin, V. L. Belenky, J. R. Paulling, K. M. Weems, and W. M. Lin, "Criteria for parametric roll of large containeships in longitudinal seas," Transactions of SNAME, vol. 112, 2004.

[16] K. J. Spyrou, I. Tigkas, G. Scanferla, N. Pallikaropoulos, and N. Themelis, "Prediction potential of the parametric rolling behaviour of a post-panamax container ship," Ocean Engineering, vol. 35, pp. 12351244, 2008.

[17] N. Umeda, H. Hashimoto, S. Minegaki, and A. Matsuda, "An investigation of different methods for the prevention of parametric rolling," Journal of Marine Science and Technology, vol. 13, pp. 16-23, 2008.

[18] M. A. S. Neves and C. A. Rodriguez, "A coupled third order model of roll parametric resonance," in Maritime Transportation and Exploitation of Ocean and Coastal Resources, C. G. Soares, Y. Garbatov, and N. Fonseca, Eds. Taylor \& Francis, 2005, pp. 243-253.

[19] —, "On unstable ship motions resulting from strong non-linear coupling," Ocean Engineering, vol. 33, pp. 1853-1883, 2006.

[20] T. I. Fossen and H. Nijmeijer, Eds., Parametric Resonance in Dynamical Systems. Springer, 2012.

[21] A. Rosén, M. Huss, and M. Palmquist, Experience from Parametric Rolling of Ships. Springer, 2012, ch. 8 in Parametric Resonance in Dynamical Systems (eds: Fossen and Nijmeijer), pp. 147-166.

[22] J. J. Jensen, Probability of Parametric Roll in Random Seaways. Springer, 2012, ch. 5 in Parametric Resonance in Dynamical Systems (eds: Fossen and Nijmeijer), pp. 91-107.

[23] R. Galeazzi, M. Blanke, and N. K. Poulsen, Detection of Parametric Roll of Ships. Springer, 2012, ch. 2 in Parametric Resonance in Dynamical Systems (eds: Fossen and Nijmeijer), pp. 17-45.

[24] C. Holden, T. Perez, and T. I. Fossen, "Frequency-motivated observer design for the prediction of parametric roll resonance," in Proceedings of the 7th IFAC Conference on Control Applications in Marine Systems, 2007.

[25] L. S. McCue and G. Bulian, "A numerical feasibility study of a parametric roll advance warning system," Journal of Offshore Mechanics and Arctic Engineering, vol. 129, pp. 165-175, 2007.

[26] R. Galeazzi, M. Blanke, and N. K. Poulsen, "Detection of parametric roll resonance on ships from indication of nonlinear energy flow," in Proceedings 7th IFAC Symp. on Fault Detection, Supervision and Safety of Technical Processes, 2009.

[27] — "Parametric roll resonance detection using phase correlation and log-likelihood testing techniques," in Proceedings of the 8th IFAC International Conference on Manoeuvring and Control of Marine Craft, 2009.

[28] S. M. Carmel, "Study of parametric rolling event on a panamax container vessel," Journal of the Transportation Research Board, vol. 1963, pp. 56-63, 2006.

[29] S. Ginsberg. (1998, November) Lawsuits rock APL's boat - Cargo goes overboard; insurance lawyers surface. San Francisco Business Times. [Online]. Available: http://www.bizjournals.com/sanfrancisco/ stories/1998/11/23/story1.html

[30] J. N. Newman, Marine Hydrodynamics. MIT Press, 1977, iSBN 0262140268.

[31] C. Holden, R. Galeazzi, C. Rodríguez, T. Perez, T. I. Fossen, M. Blanke, and M. A. S. Neves, "Nonlinear container ship model for the study of parametric roll resonance," Modeling, Identification and Control, vol. 28, pp. 87-113, 2007.

[32] A. H. Nayfeh and D. T. Mook, Nonlinear Oscillations. WILEY-VCH, 2004.

[33] R. Grimshaw, Nonlinear Ordinary Differential Equations. CRC Press, 1993.

[34] I. G. Oh, A. H. Nayfeh, and D. T. Mook, "A theoretical and experimental investigations of indirectly excited roll motion in ships," Philosophical Transactions: Mathematical, Physical and Engineering Sciences, vol. 358, pp. 1853-1881, 2000.

[35] A. Tondl, T. Ruijgrok, F. Verhulst, and R. Nabergoj, Autoparametric Resonance in Mechanical Systems. Cambridge University Press, 2000.
[36] SNAME, "Nomenclature for treating the motion of a submerged body through a fluid," Society of Naval Architects and Marine Engineers, New York, USA, Tech. Rep., 1950.

[37] N. Balakrishnan and M. Kateri, "On the maximum likelihood estimation of parameters of weibull distribution based on complete and censored data," Statistics and Probability Letters, vol. 78, pp. 2971-2975, 2008.

[38] S. M. Kay, Fundamental of Statistical Signal Processing vol II: Detection Theory. Prentice Hall, 1998.

[39] V. L. Belenky, K. M. Weems, W. M. Lin, and J. R. Paulling, "Probabilistic analysis of roll parametric resonance in head seas," in Proceedings of the 8th International Conference on Stability of Ships and Ocean Vehicles, 2003.

[40] R. Galeazzi, M. Blanke, and N. K. Poulsen, "Prediction of resonant oscillation, EP09157857, Filed 2009.” Denmark Patent EP 09157857, April, 2009.

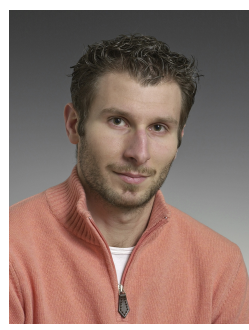

Roberto Galeazzi (S'06-M'10) received the M.Sc. degree from Univesità Politecnica delle Marche, Italy, in 2005, and the Ph.D. degree from the Technical University of Denmark in 2010, both in electrical engineering.

He was with the Centre for Ships and Ocean Structures, Norwegian University of Science and Technology, as Post-doc in 2009-2010, with DTU Electrical Engineering as Post-doc in 2010-2011. He is currently Assistant Professor at DTU Electrical Engineering, Technical University of Denmark. His research focuses on nonlinear systems analysis and control, and statistical change detection.

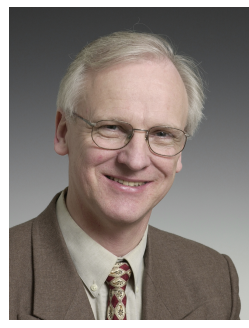

Mogens Blanke (M 1974, SM 1985), received the MScEE degree in 1974 and the PhD degree in 1982 from the Technical University of Denmark, DTU.

He was Systems Analyst with the European Space Agency 1975-76, with DTU 1977-84, was Head of Division at Lyngsø Marine 1985-89, Professor at Aalborg University 1990-99. He is now (2000 -) Professor in Automation and Control at DTU and since 2005 also Adjunct Professor at CeSOS at the Norwegian University of Science and Technology. His research interests are automation and control in general and diagnosis and fault tolerant control as area of special focus Application areas include marine systems and autonomous vehicles.

Prof. Blanke has held various positions in the International Federation of Automatic Control, including founding member and first Chair of the Technical Committee on Marine Systems, Coordinating Committee Chair and Member of Council. He is Assoc. Editor for Control Engineering Practice and Technical Editor for IEEE Transactions of Aerospace and Electronic Systems.

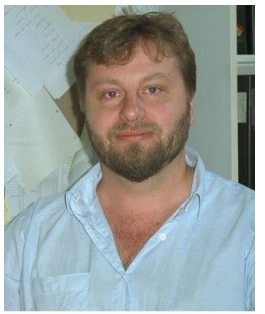

Niels Kjølstad Poulsen (M 1996, SM 2001) received his M.Sc. and Ph.D. degrees in electrical engineering from the Institute of Mathematical Statistics and Operations Research (IMSOR), the Technical University of Denmark, in 1981 and 1984 , respectively.

He was Post-doc at IMSOR in 1984-1986 and Assistant Professor in 1986-1990. Since 1990 he has held a position as Associate Professor at Department of Informatics and Mathematical Modelling, Technical University of Denmark. His primary research interests are within stochastic control theory, system identification and fault diagnosis. The application areas are modelling and control wind turbines and Robotics.

Dr. Poulsen has been Member of two IFAC technical committees and the chairman (1998-2002) for IEEE joint chapter on Control Systems/Robotics and Automation, IEEE Denmark Section. 